\title{
Novel directed synthesis of functionalized pyrazole derivatives via regioselective solvent-free thiylation of 3-alkenylpyrazoles with arenethiols
}

\author{
Galina G. Levkovskaya, Elena V. Rudyakova, Valentina A. Kobelevskaya, \\ Aleksandr V. Popov, * and Igor B. Rozentsveig \\ A. E. Favorsky Irkutsk Institute of Chemistry Russian Academy of Sciences, Siberian Branch, \\ Favorsky Str. 1, Irkutsk 664 033, Russia \\ E-mail:popov@irioch.irk.ru
}

DOI: http://dx.doi.org/10.3998/ark.5550190.p009.383

\begin{abstract}
Unprecedented easy (3 min., room temperature) regioselective addition of thiols to the endocyclic double bond of 3-vinyl-, prop-1-enyl- and isopropenyl, including those with bulky substituents in the position 1 of the pyrazole ring, has been found for the reaction of arenethioles with 3-alkenyl-5-chloropyrazoles. The reaction proceeds under solvent-free and catalyst-free conditions to afford 3-[(2-arylsulfanyl)alkyl]-5-chloropyrazoles in excellent yields. High rate of the process is likely due to protonation of the "pyridine" nitrogen atom of the pyrazole ring to give a pyrazolium ion which activates the vinyl group towards nucleophilic attack by the simultaneously formed arylthiolate anions.
\end{abstract}

Keywords: Solvent-free reactions, catalyst-free reactions, $C$-alkenylpyrazoles, arenethiols, thiylation

\section{Introduction}

Pyrazole and its derivatives attract the attention of the chemical community mainly on account of the wide spectrum of their pharmacological activities, ${ }^{1-6}$ including antibacterial, ${ }^{7}$ antidepressant, ${ }^{8}$ antiinflammatory, ${ }^{9}$ antitumor, ${ }^{10}$ etc. They are widely used as agrochemicals. ${ }^{11-14}$ Pyrazole derivatives exhibiting acaricidal action and applied in modern practical disinfection and decontamination are of special importance. ${ }^{15-17}$

In recent years, increasing interest has been focused on polypyrazole constructs which are promising ligands. ${ }^{18}$ Some pyrazole ligands can be used in transition metal-catalyzed reactions. ${ }^{19}$ 
Pyrazoles are used in supramolecular ${ }^{20}$ and polymer chemistry, ${ }^{21}$ in the food industry, as cosmetic colorings ${ }^{22}$ and UV stabilizers ${ }^{23}$ and some possess liquid crystal properties. ${ }^{24,25}$ They are also applied in the design of complexes with unusual magnetic properties. ${ }^{26,27}$

In the search for novel pyazoles as building blocks for the synthesis of biologically active and technologically useful substances, ${ }^{1-6}$ the development of new methods for the preparation of functionalized pyrazoles and the synthesis of new pyrazole derivatives present an urgent challenge.

Alkenylpyrazoles are valuable and promising building blocks for the synthesis of diverse pyrazole derivatives including pyrazole-containing multi-nuclear and annelated heterocyclic systems. ${ }^{1-6,28-39}$ The chemical transformations of alkenylpyrazoles are still poorly understood. It is known that $N$-alkenyl- and some 4-alkenylpyrazoles can participate in polymerization, ${ }^{21,29}$ complex formation, ${ }^{30,31}$ thiylation, ${ }^{32}$ and cycloaddition. ${ }^{33-35}$ Imidoalkylation of 1-, 3-, 4-, and 5vinylpyrazoles with $N$-(2,2,2-trichloroethylidene)ethoxycarbonylamines is also reported r $^{34,36}$ Chemical transformations of the difficult-to-access 3-alkenylpyrazoles ${ }^{36-38}$ and 3-alkenyl-5chloropyrazoles $^{28}$ are even less studied and only very few reactions with oxygen and sulphur nucleophiles have been published. ${ }^{39,40}$

We have recently developed methodology for the preparation of hitherto unknown 3alkenylpyrazoles, ${ }^{28}$ possible building blocks for the directed synthesis of substances and materials for advanced technologies, from the corresponding 3-haloalkyl pyrazoles. The aim of the present work is to study of chemical behavior of 3-alkenylpyrazoles in the reactions with aromatic and aliphatic thiols.

The reactions of 1-vinyl- and 1-iso-propenylpyrazoles with thiols have been reported. ${ }^{32,41}$ As is known, ${ }^{32}$ 1-vinylpyrazoles react with thiols via both ionic (in the presence of $\mathrm{S}, \mathrm{SO}_{2}$, $\mathrm{BF}_{3} \cdot \mathrm{Et}_{2} \mathrm{O}, p$-toluenesulfonic acid) and free radical mechanisms to afford $\alpha$ - and $\beta$-adducts.

The reaction turns out to be non-chemoselective. Radical inhibitors (benzoquinone, hydroquinone, sulfur) did not exclude formation of $\beta$-adducts. ${ }^{32}$ The yield of $\alpha$-adducts in the reaction of 1-vinylpyrazole with thiols under ionic conditions did not exceed 53\%. Upon heating from $20^{\circ} \mathrm{C}$ to $80^{\circ} \mathrm{C}$ and in the presence of radical initiators (photoirradiation or AIBN), the process was considerably accelerated (from $24 \mathrm{~h}$ to $10 \mathrm{~min}$ ), but without improving the selectivity.

1-Isopropenylpyrazole reacts with EtSH in the presence of AIBN at $65{ }^{\circ} \mathrm{C}$ for $3.5 \mathrm{~h}$ with total conversion of the initial pyrazole. The yield of the target $\beta$-adduct was $75 \%$. ${ }^{41}$ The reaction with thiophenol under the same conditions proceeds with low conversion of the 1-alkenylpyrazole (42\%) to give the adduct in $74 \%$ yield (based on the reacted alkenylpyrazole). At higher temperatures conversion of the isopropenylpyrazole increases, but the yield of the target product dramatically drops due to side reactions. ${ }^{41}$ There are no essential differences in the reactions of 1-alkenylpyrazoles with aromatic or aliphatic thiols. ${ }^{32,41}$

Similar reactions of 1-vinylpyrroles, 1-vinylimidazoles, and 1-vinylindoles ${ }^{42,43}$ with thiols also occur in the presence of AIBN. Aromatic thiols react without catalyst over a longer time 
(18-25 h, 70-80 ${ }^{\circ} \mathrm{C}$ ) to deliver $\beta$-adducts. ${ }^{42,43} \mathrm{~N}$-Vinylpyrroles react with thiophenols giving rise to both $\beta$ - $(80 \%)$ and $\alpha$-adducts $(20 \%){ }^{43}$

Addition of arenethiols to electron deficient double bonds of $\alpha, \beta$-unsaturated carbonyl compounds or nitriles are catalyzed by sodium borate ${ }^{44}$ or lithium perchlorate. ${ }^{45}$

We have developed a synthesis of the so far scarcely accessible 3-alkenylpyrazoles ${ }^{37,38}$ and hitherto unknown 3-alkenyl-5-chloropyrazoles. ${ }^{28}$ This enables pioneering data on the chemical properties of these compounds to be obtained, and to allow comparison of their structural peculiarities and reactivity with those the 1-alkenylpyrazoles.

\section{Results and Discussion}

In the present work a series of 3-vinyl-, 3-propenyl- and 3-isopropenyl-5-chloropyrazoles (4a-g) from synthesized 2,2-dichlorovinyl-[1-chloro(bromo)alkyl]ketones and 1,1-dimethylhydrazines or alkylhydrazines using the methodology developed recently. ${ }^{28}$ (Scheme 1)

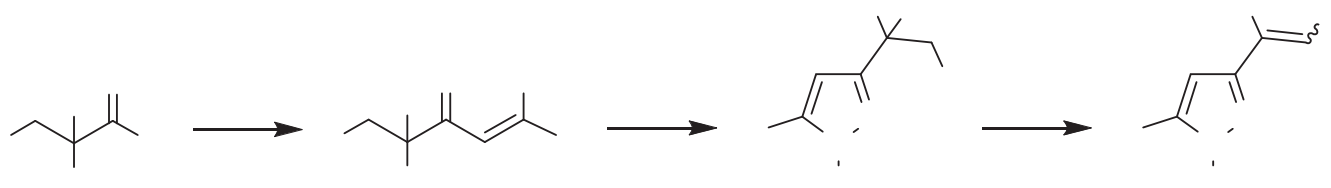

$36-50 \%$

Scheme 1. Reagents, intermediate products and resulting 3-(alkenyl)pyrazoles 4.

Yields of the target pyrazoles calculated from starting acyl chlorides $\mathbf{1}$, and the synthesis of 3-vinylpyrazoles $\mathbf{4 a}, \mathbf{b}$ were described in our previous publication ${ }^{28}$ pyrazole $\mathbf{4 g}$ was obtained as a mixture of $E$-and $Z$-isomers in a 4:1 ratio. The reactions of 2,2-dichlorovinylketone 2a with dimethylhydrazine and benzylhydrazine, synthesis of intermediate 3-(1-chloroethyl)pyrazoles 3a,b and final 3-vinylpyrazoles $\mathbf{4 a}, \mathbf{b}$ were also described in the previous paper. ${ }^{28}$ The reactions of dichlorovinylketones $\mathbf{2 a , b}$ with other hydrazines, the reactions of ketones $\mathbf{2} \mathbf{c}, \mathbf{d}$ with dimethylhydrazine, and the synthesis of pyrazole derivatives $\mathbf{3 c - j}$ and $\mathbf{4 c - g}$ are presented here for the first time.

According to the methodology, ${ }^{28} \alpha$-chloroacyl chlorides 1 were involved into the reaction with vinylidene dichloride in the presence of $\mathrm{AlCl}_{3}$. For $\alpha$-bromopropionyl chloride $\mathbf{1 b}$, the 
substitution of bromine by a chlorine atom in $\alpha$-position under the action of $\mathrm{AlCl}_{3}$ took place and mixtures of intermediate ( $\alpha$-haloalkyl)(dichlorovinyl)ketones $\mathbf{2 a}, \mathbf{b}$ were produced. In these cases ketones $\mathbf{2 a , b}$ were introduced into further reactions with hydrazines without isolation in pure form to give mixtures of corresponding intermediate 3-(1-haloethyl)pyrazoles 3c,d, 3e,f and $\mathbf{3 g}, \mathbf{h}$, which were transformed without separation into the final 3-vinylpyrazoles $\mathbf{4 c - e}$.

The systematic investigations of the reactions between 3-alkenylpyrazoles and aromatic or aliphatic thiols showed that arenethiols instantly reacted with 3-alkenylpyrazoles $\mathbf{4 a - g}$ to afford 3-[(2-arylsulfanyl)alkyl]pyrazoles in quantitative yields (Table 1). The process was carried out under solvent-free and catalyst-free conditions. 3-Vinyl-, 3-propenyl and 3-isopropenylpyrazoles 4a-g, all reacted in a couple of minutes under similar conditions to give $\beta$-adducts. Side-products including $\alpha$-adducts or oligomers (polyvinylpyrazoles) were not detected.

Table 1. Reaction ${ }^{a}$ of 3-alkenylpyrazoles 4 with thiols 5 ; adducts 6
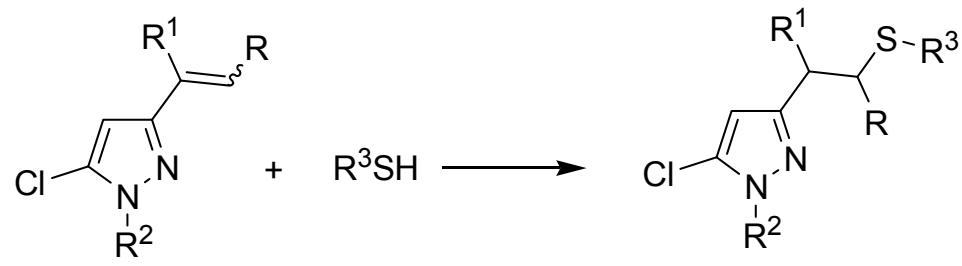

4

\begin{tabular}{|c|c|c|c|c|c|c|c|c|}
\hline \multirow{2}{*}{ Entry } & \multicolumn{4}{|c|}{ 3-Alkenylpyrazoles 4} & \multicolumn{2}{|c|}{ Thiols 5} & \multicolumn{2}{|c|}{ Adducts 6} \\
\hline & & $\mathrm{R}$ & $\mathrm{R}^{1}$ & $\mathrm{R}^{2}$ & & $\mathrm{R}^{3}$ & & eld, \% \\
\hline 1 & $4 a$ & $\mathrm{H}$ & $\mathrm{H}$ & $\mathrm{Me}$ & $5 \mathbf{a}$ & $\mathrm{Ph}$ & 6aa & 86 \\
\hline 2 & $4 \mathbf{a}$ & $\mathrm{H}$ & $\mathrm{H}$ & $\mathrm{Me}$ & $5 \mathbf{b}$ & $4-\mathrm{ClC}_{6} \mathrm{H}_{4}$ & 6ab & 94 \\
\hline 3 & $4 \mathbf{a}$ & $\mathrm{H}$ & $\mathrm{H}$ & $\mathrm{Me}$ & $5 c$ & $4-\mathrm{BrC}_{6} \mathrm{H}_{4}$ & 6ac & 92 \\
\hline 4 & $4 a$ & $\mathrm{H}$ & $\mathrm{H}$ & $\mathrm{Me}$ & $5 d$ & $4-\mathrm{MeC}_{6} \mathrm{H}_{4}$ & 6ad & 90 \\
\hline 5 & $4 b$ & $\mathrm{H}$ & $\mathrm{H}$ & $\mathrm{Bn}$ & $5 \mathbf{a}$ & $\mathrm{Ph}$ & $6 \mathbf{b a}$ & 89 \\
\hline 6 & $4 b$ & $\mathrm{H}$ & $\mathrm{H}$ & $\mathrm{Bn}$ & $5 \mathbf{b}$ & $4-\mathrm{ClC}_{6} \mathrm{H}_{4}$ & $6 \mathbf{b b}$ & 94 \\
\hline 7 & $4 b$ & $\mathrm{H}$ & $\mathrm{H}$ & $\mathrm{Bn}$ & $5 c$ & $4-\mathrm{BrC}_{6} \mathrm{H}_{4}$ & 6bc & 93 \\
\hline 8 & $4 c$ & $\mathrm{H}$ & $\mathrm{H}$ & $i$-Pr & $5 \mathbf{a}$ & $\mathrm{Ph}$ & 6ca & 95 \\
\hline 9 & $4 c$ & $\mathrm{H}$ & $\mathrm{H}$ & $i-\operatorname{Pr}$ & $5 b$ & $4-\mathrm{ClC}_{6} \mathrm{H}_{4}$ & $6 \mathrm{cb}$ & 95 \\
\hline 10 & $4 d$ & $\mathrm{H}$ & $\mathrm{H}$ & $i$-Am & $5 a$ & $\mathrm{Ph}$ & 6da & 98 \\
\hline 11 & $4 e$ & $\mathrm{H}$ & $\mathrm{H}$ & $n$-Ht & $5 \mathbf{a}$ & $\mathrm{Ph}$ & 6ea & 98 \\
\hline 12 & $4 e$ & $\mathrm{H}$ & $\mathrm{H}$ & $n-\mathrm{Ht}$ & $5 b$ & $4-\mathrm{ClC}_{6} \mathrm{H}_{4}$ & $6 e b$ & 95 \\
\hline 13 & $4 f$ & $\mathrm{H}$ & $\mathrm{Me}$ & $\mathrm{Me}$ & $5 \mathbf{a}$ & $\mathrm{Ph}$ & $6 \mathrm{fa}$ & 97 \\
\hline 14 & $4 f$ & $\mathrm{H}$ & $\mathrm{Me}$ & $\mathrm{Me}$ & $5 b$ & $4-\mathrm{ClC}_{6} \mathrm{H}_{4}$ & $6 \mathrm{fb}$ & 98 \\
\hline 15 & $4 g$ & $\mathrm{Me}$ & $\mathrm{H}$ & $\mathrm{Me}$ & $5 b$ & $4-\mathrm{ClC}_{6} \mathrm{H}_{4}$ & $6 \mathrm{gb}$ & 96 \\
\hline $16^{\mathrm{a}}$ & $4 a$ & $\mathrm{H}$ & $\mathrm{H}$ & $\mathrm{Me}$ & $5 e$ & $n-\operatorname{Pr}$ & 6ae & 0 \\
\hline $17^{\mathrm{a}}$ & $4 \mathbf{a}$ & $\mathrm{H}$ & $\mathrm{H}$ & $\mathrm{Me}$ & $5 f$ & $\mathrm{Bn}$ & 6af & 0 \\
\hline
\end{tabular}

${ }^{a}$ Reaction conditions: 4 (1 mmol), thiol 5 (1 mmol), solvent-free, r.t., 3 min. 
In contrast to aromatic thiols, alkanetiols did not react with 3-alkenyl-5-chloropyrazoles under the same conditions. For example, 1-methyl-3-vinyl-5-chloropyrazole (4a) did not give adducts with propylmercaptan or benzylmercaptan (Table 1, Entries 16, 17).

The structures of sulfur-containing pyrazoles 6 were proved by IR and NMR spectra, the composition was confirmed by the data of elemental analysis.

In the ${ }^{1} \mathrm{H}$ and ${ }^{13} \mathrm{C}$ NMR spectra of products 6 signals of the alkenyl groups (typical for the starting 3-alkenylpyrazoles 4 are absent, while signals of organylsulfanylethyl moieties with characteristic shifts are observed. A feature of the ${ }^{1} \mathrm{H}$ NMR spectra of compounds $\mathbf{6} \mathbf{f a}, \mathbf{6} \mathbf{f b}$ is the presence of diastereotropic protons of the $\mathrm{CH}_{2} \mathrm{SR}$ and $\mathrm{CH}_{2}$ groups which are non-equivalent and show as multiplets with different chemical shifts.

Furthermore, we directly synthesized 3-(1-phenylsulfanylethyl)pyrazole 7 which is isomeric and non-identical with compound 6ca (Scheme 2).
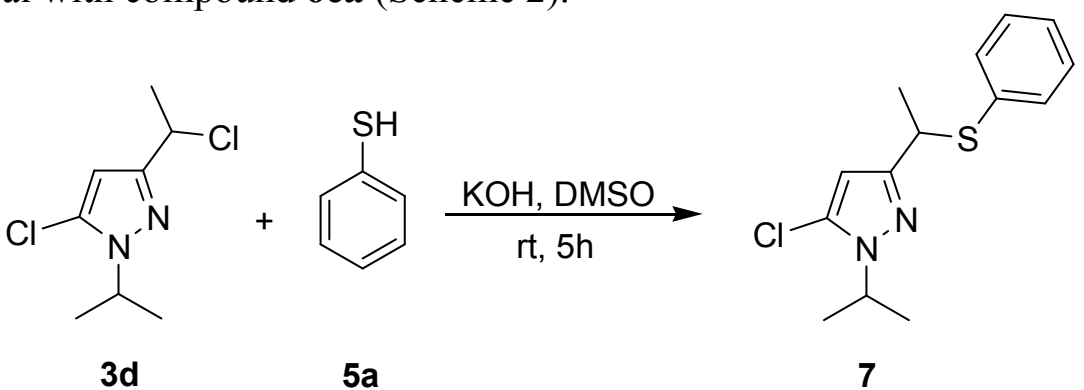

Scheme 2. Reaction of 3-( $\alpha$-chloroethyl)pyrazole 3d with thiol 5a.

It was shown previously ${ }^{28}$ that 3 -( $\alpha$-chloroalkyl)pyrazoles do not give the corresponding 3alkenylpyrazoles under the action of $\mathrm{KOH}$ in DMSO at r.t. This fact allows suggesting, that reaction in Scheme 2, proceeds by nucleophilic substitution of the $\alpha$-chlorine atom by the thiol without dehydrochlorination-addition stages.

Comparison of NMR spectra for isomers 7 and $\mathbf{6 c a}$ unambiguously proves the direction of reaction of alkenylpyrazoles 4 with arenethiols $\mathbf{6}$ and the formation only of $\beta$-adducts.

\section{Conclusions}

In conclusion, an efficient one-pot method for the synthesis of hitherto unknown pyrazoles via the atom economical reaction between 3-alkenylpyrazoles and arenethiols has been developed. The synthesized pyrazoles combine two biologically relevant fragments in their structures (the azole ring and the sulfanyl group) and represent promising building blocks for drug design, pesticides, and other useful products.

The reaction of arenethiols with 3-alkenylpyrazoles is chemo- and regio-selective. The high rate of the process is likely due to protonation of the "pyridine" nitrogen atom of the pyrazole ring to give a pyrazolium fragment which activates the vinyl group towards nucleophilic attack by the 
simultaneously formed arylthiolate anions (more nucleophilic than neutral thiols) thus generated are easy added to the activated alkenyl group.

Interestingly, the studied reaction does not represent classical electrophilic addition of arenethiols to the double bond. Apparently it is an autocatalytic process which accompanied by electrophilic assistance to nucleophilic addition. This reaction allows synthesis of new derivatives of the pyrazole. Besides, it can ensure a promising route to other pyrazole derivatives through the activation of alkenyl groups of 3-alkenylpyrazoles by mild protonation with subsequent nucleophilic addition of the reagents to the double bond.

\section{Experimental Section}

General. The ${ }^{1} \mathrm{H}$ and ${ }^{13} \mathrm{C}$ NMR spectra were recorded on a Bruker DPX-400 spectrometer (400.13 and $100.61 \mathrm{MHz}$, respectively) in $\mathrm{CDCl}_{3}$. Chemical shifts $(\delta)$ in ppm are reported with use of the residual chloroform $\left(7.25\right.$ for ${ }^{1} \mathrm{H}$ and 77.20 for $\left.{ }^{13} \mathrm{C}\right)$ as internal standards. IR spectra were recorded on a Bruker Vertex 70 spectrometer in the region of $400-4000 \mathrm{~cm}^{-1}$. MS analyses were recorded on a Shimadzu GCMS-QP5050A instrument (ionization potential $70 \mathrm{eV}$ ). Monitoring of the reaction progress and the analysis of the resulting liquid products were performed on a chromatograph LHM 80-MD-2 (column $3 \times 2000 \mathrm{~mm}$, liquid phase is DC-550, $5 \%$ on the recording medium Chromaton N-AW-HMDS, linear programming mode temperature $12 \mathrm{deg} / \mathrm{min}$, carrier gas helium). The elemental analyses were carried out on Flash EA 1112 elemental analyzer. MW activation was carried out with an Anton Paar Monowave 300 oven at the temperature control. Commercially available $\alpha$-haloacylchlorides $\mathbf{1 a - d}$, thiols $\mathbf{5}$ and alkyl hydrazines were used. Alkyl hydrazines were distilled before reaction.

Synthesis of 2,2-dichlorovinylketones 2a-d, 3-(1-chloroethyl)pyrazoles 3a,b, and 3-vinylpyrazoles $\mathbf{4 a}, \mathbf{b}$ was presented in a previous paper. ${ }^{28}$ Pyrazole derivatives $\mathbf{3 c}-\mathbf{j}$ and $\mathbf{4 c - g}$ are described here for the first time.

\section{General procedure for the synthesis of pyrazole derivatives $3 \mathrm{c}-\mathrm{h}$ and $4 \mathrm{c}-\mathrm{e}$}

A mixture of triethylamine $(50 \mathrm{mmol}, 5.05 \mathrm{~g})$ and the corresponding alkylhydrazine $(50 \mathrm{mmol})$ was added dropwise to a of dichlorovinylketones $\mathbf{2 a}$ or $\mathbf{2 b}$ or to a mixture of dichlorovinylketones $\mathbf{2 a}$ and $\mathbf{2 b}(50 \mathrm{mmol})^{28}$ in diethyl ether $(150 \mathrm{~mL})$ for $20 \mathrm{~min}$. After completion of the exotherm, the reaction mixture was stirred for $5 \mathrm{~h}$ and poured into water (150 $\mathrm{mL})$. The organic layer was separated and the water layer was extracted with diethyl ether $(3 \times$ $50 \mathrm{~mL}$ ). The organic layer combined with the extract was dried over $\mathrm{CaCl}_{2}$, filtered off. After evaporating of diethyl ether 3-(1-chloroethyl)pyrazoles 3d, 3f, 3h or mixtures of 3-(1haloethyl)pyrazoles $\mathbf{3 c}$ and $\mathbf{3 d}, \mathbf{3 e}$ and $\mathbf{3 f}, \mathbf{3 g}$ and $\mathbf{3 h}$ or mixtures of 3-(1-bromo-1-methylethyl)5-chloro-1-methyl-1H-pyrazole (3i) and 5-chloro-1-methyl-3-(1-methylethenyl)-1H-pyrazole (4f) were used for further dehydrohalogenation without additional purification. The corresponding 3-(1-chloroethyl pyrazoles or mixtures of 3-(1-haloethyl)pyrazoles or mixture 
pyrazoles (3i) and (4f) in DMF $(20 \mathrm{~mL})$ was exposed under MW irradiation $\left(150^{\circ} \mathrm{C}\right)$ for $10-20$ min. Then the reaction mass was diluted with water $(200 \mathrm{~mL})$ and extracted with diethyl ether (3 $\times 50 \mathrm{~mL}$ ). The extract was dried over $\mathrm{MgSO}_{4}$ and filtered off. Diethyl ether was evaporated off and the target 3-alkenylpyrazoles (4c-f) colourless liquid were distilled in vacuum.

Mixture of 3-(1-bromoethyl)-5-chloro-1-(1-methylethyl)-1H-pyrazole (3c) and 5-chloro-3(1-chloroethyl)-1-(1-methylethyl)-1H-pyrazole (3d). Colourless liquid, bp 112-118 ${ }^{\circ} \mathrm{C}(25 \mathrm{~mm}$ $\mathrm{Hg}),(9.7 \mathrm{~g}, 3 \mathbf{c} 8.2 \mathrm{~g}, 77 \%$; 3d $1.5 \mathrm{~g}, 14.5 \%)$, ratio 3c (88\%) : 3d (12\%), is determined from the intensity signals of the protons of the groups of $\mathrm{CHCl}$ and $\mathrm{CHBr}$ in the ${ }^{1} \mathrm{H}$ NMR spectrum; IR $\left(v_{\max }, \mathrm{cm}^{-1}\right)$ : 3c and 3d: $3131\left(=\mathrm{C}-\mathrm{H}_{\mathrm{Pyr}}\right), 2950,2919,2855\left(\mathrm{CH}_{\mathrm{Alk}}\right), 1601,1515(\mathrm{C}=\mathrm{C}, \mathrm{C}=\mathrm{N})$.

3c. ${ }^{1} \mathrm{H}$ NMR $\left(400.13 \mathrm{MHz}, \mathrm{CDCl}_{3}\right): \delta_{\mathrm{H}} 6.25\left(\mathrm{~s}, 1 \mathrm{H}, \mathrm{H}^{4}\right), 5.19$ (q, J $\left.6.9 \mathrm{~Hz}, 1 \mathrm{H}, \mathrm{CHBr}\right), 4.60$ (spt, $J 6.6 \mathrm{~Hz}, 1 \mathrm{H}, \mathrm{NCH}), 1.98\left(\mathrm{~d}, J 6.9 \mathrm{~Hz}, 3 \mathrm{H}, \mathrm{CH}_{3}\right), 1.42$ (d, J 6.6 Hz, 6H, $\left.\mathrm{CH}_{3}\right)$. MS (EI, $\left.70 \mathrm{eV}\right)$, $\mathrm{m} / \mathrm{z}(\%): 250\left(\mathrm{M}^{+}, 2\right), 235$ (9), 208 (4), 193 (35), 178 (62), 136(100).

3d. ${ }^{1} \mathrm{H}$ NMR (400.13 MHz, $\left.\mathrm{CDCl}_{3}\right): \delta_{\mathrm{H}} 6.23\left(\mathrm{~s}, 1 \mathrm{H}, \mathrm{H}^{4}\right.$ ), 5.18 (q, J $\left.6.9 \mathrm{~Hz}, 1 \mathrm{H}, \mathrm{CHCl}\right), 4.35$ (spt, $J 6.8 \mathrm{~Hz}, 1 \mathrm{H}, \mathrm{NCH}), 1.82\left(\mathrm{~d}, J 6.9 \mathrm{~Hz}, 3 \mathrm{H}, \mathrm{CH}_{3}\right), 1.44\left(\mathrm{~d}, J 6.8 \mathrm{~Hz}, 6 \mathrm{H}, \mathrm{CH}_{3}\right) .{ }^{13} \mathrm{C} \mathrm{NMR}(100.61$ $\left.\mathrm{MHz}, \mathrm{CDCl}_{3}\right): \delta_{\mathrm{C}} 152.8\left(\mathrm{C}^{3}\right), 125.8\left(\mathrm{C}^{5}\right), 101.7\left(\mathrm{C}^{4}\right), 58.3(\mathrm{CHCl}), 52.7\left(\mathrm{NCH}_{2}\right), 50.1\left(\mathrm{CH}_{2}\right)$, 24.6 $\left(\mathrm{CH}_{3}\right), 21.6\left(\mathrm{CH}_{3}\right)$. MS (EI, $\left.70 \mathrm{eV}\right), \mathrm{m} / \mathrm{z}(\%): 206\left(\mathrm{M}^{+}, 15\right), 171(50), 129(100)$.

5-Chloro-3-(1-chloroethyl)-1-(1-methylethyl)-1H-pyrazole (3d). Synthesized from ketones $\mathbf{2 a}$ and 1-methylethylhydrazine. Colourless liquid $(7.9 \mathrm{~g}, 76 \%)$, bp $112-113^{\circ} \mathrm{C}(24 \mathrm{~mm} \mathrm{Hg})$, IR $\left(v_{\max }, \mathrm{cm}^{-1}\right): 3131\left(=\mathrm{C}-\mathrm{H}_{\text {Pyr }}\right), 2950,2919,2855\left(\mathrm{CH}_{\mathrm{Alk}}\right), 1601,1515(\mathrm{C}=\mathrm{C}, \mathrm{C}=\mathrm{N}) .{ }^{1} \mathrm{H}$ NMR $\left(400.13 \mathrm{MHz}, \mathrm{CDCl}_{3}\right): \delta_{\mathrm{H}} 6.25\left(\mathrm{~s}, 1 \mathrm{H}, \mathrm{H}^{4}\right), 5.10(\mathrm{q}, J=6.9 \mathrm{~Hz}, 1 \mathrm{H}, \mathrm{CHCl}), 4.61(\mathrm{spt}, J=6.7 \mathrm{~Hz}$, $1 \mathrm{H}, \mathrm{NCH}), 1.82\left(\mathrm{~d}, J=6.9 \mathrm{~Hz}, 3 \mathrm{H}, \mathrm{CH}_{3}\right), 1.46\left(\mathrm{~d}, J=6.7 \mathrm{~Hz}, 6 \mathrm{H}, 2 \mathrm{CH}_{3}\right) . \mathrm{MS}(\mathrm{EI}, 70 \mathrm{eV}), \mathrm{m} / \mathrm{z}(\%)$ : $206\left(\mathrm{M}^{+}, 15\right), 171$ (50), 129 (100). Elemental anal. Calcd for $\mathrm{C}_{8} \mathrm{H}_{12} \mathrm{Cl}_{2} \mathrm{~N}_{2}: \mathrm{C}, 46.40 ; \mathrm{H} \mathrm{5.84;} \mathrm{Cl}$, 34.24; N, 13.53. Found: C, 46.47; H, 5.77; Cl, 34.30; N, 13.39.

Mixture of 3-(1-bromoethyl)-5-chloro-1-(3-methylbutyl)-1 $\mathrm{H}$-pyrazole (3e) and 5-chloro-3(1-chloroethyl)-1-(3-methylbutyl)-1H-pyrazole (3f). Colourless liquid (12.5 g: 3e 10.3 g, 67\%; 3f. $2.2 \mathrm{~g}, 16.8 \%)$, bp $145-147{ }^{\circ} \mathrm{C}(25 \mathrm{~mm} \mathrm{Hg})$, ratio $3 \mathbf{e}(63 \%)$ : $3 \mathbf{f}(37 \%)$ is determined from the intensity signals of the protons of the groups of $\mathrm{CHCl}$ and $\mathrm{CHBr}$ in the $1 \mathrm{H} \mathrm{NMR} \mathrm{spectrum;} \mathrm{IR}$ $\left(v_{\max }, \mathrm{cm}^{-1}\right)$ : 3e and 3f: $3132\left(=\mathrm{C}-\mathrm{H}_{\mathrm{Pyr}}\right), 2952,2920,2858\left(\mathrm{CH}_{\mathrm{Alk}}\right), 1603,1517(\mathrm{C}=\mathrm{C}, \mathrm{C}=\mathrm{N})$.

3e. ${ }^{1} \mathrm{H}$ NMR $\left(400.13 \mathrm{MHz}, \mathrm{CDCl}_{3}\right): \delta_{\mathrm{H}} 6.28\left(\mathrm{~s}, 1 \mathrm{H}, \mathrm{H}^{4}\right), 5.19(\mathrm{q}, J 6.9 \mathrm{~Hz}, 1 \mathrm{H}, \mathrm{CHBr}), 4.09$ (t, $J$ $\left.7.6 \mathrm{~Hz}, 2 \mathrm{H}, \mathrm{NCH}_{2}\right), 1.86\left(\mathrm{~d}, J 6.9 \mathrm{~Hz}, 3 \mathrm{H}, \mathrm{CH}_{3}\right), 1.77\left(\mathrm{~m}, 3 \mathrm{H}, \mathrm{CH}, \mathrm{CH}_{2}\right), 0.93(\mathrm{~d}, J 6.7,6 \mathrm{H}$, $\left.2 \mathrm{CH}_{3}\right)$.

3f. ${ }^{1} \mathrm{H}$ NMR (400.13 MHz, $\left.\mathrm{CDCl}_{3}\right): \delta_{\mathrm{H}} 6.28\left(\mathrm{~s}, 1 \mathrm{H}, \mathrm{H}^{4}\right), 5.12(\mathrm{q}, J 6.8 \mathrm{~Hz}, 1 \mathrm{H}, \mathrm{CHCl}), 4.16$ (q, $J$ $7.2 \mathrm{~Hz}, 2 \mathrm{H}, \mathrm{NCH}_{2}$ ) $, 1.92\left(\mathrm{~d}, J 6.8 \mathrm{~Hz}, 3 \mathrm{H}, \mathrm{CH}_{3}\right), 1.58\left(\mathrm{~m}, 3 \mathrm{H}, \mathrm{CH}, \mathrm{CH}_{2}\right), 0.95$ (d, J 6.7 Hz, 6H, $\left.2 \mathrm{CH}_{3}\right)$. ). ${ }^{13} \mathrm{C} \mathrm{NMR}\left(100.61 \mathrm{MHz}, \mathrm{CDCl}_{3}\right): \delta_{\mathrm{C}} 153.6\left(\mathrm{C}^{3}\right), 127.4\left(\mathrm{C}^{5}\right), 102.5\left(\mathrm{C}^{4}\right), 52.3(\mathrm{CHCl})$, $48.0\left(\mathrm{NCH}_{2}\right), 38,8(\mathrm{CH}), 26.01\left(\mathrm{CH}_{2}\right), 25.1\left(\mathrm{CH}_{3}\right), 22.6\left(\mathrm{CH}_{3}\right)$.

5-chloro-3-(1-chloroethyl)-1-(3-methylbutyl)-1H-pyrazole (3f). Synthesized from ketones 2a and 3-methylbutylhylhydrazine. Colourless liquid (7.3 g, $62 \%)$, bp $145-147^{\circ} \mathrm{C}(25 \mathrm{~mm} \mathrm{Hg})$. IR $\left(v_{\max }, \mathrm{cm}^{-1}\right): 3131\left(=\mathrm{C}-\mathrm{H}_{\mathrm{Pyr}}\right), 2950,2919,2855\left(\mathrm{CH}_{\mathrm{Alk}}\right), 1601,1515(\mathrm{C}=\mathrm{C}, \mathrm{C}=\mathrm{N}) .{ }^{1} \mathrm{H}$ NMR $\left(400.13 \mathrm{MHz}, \mathrm{CDCl}_{3}\right): \delta_{\mathrm{H}} 6.26\left(\mathrm{~s}, 1 \mathrm{H}, \mathrm{H}^{4}\right), 5.09$ (q, $\left.J=6.9 \mathrm{~Hz}, 1 \mathrm{H}, \mathrm{CHCl}\right), 4.10(\mathrm{~m}, J=6.8,1.3$ $\left.\mathrm{Hz}, 2 \mathrm{H}, \mathrm{NCH}_{2}\right), 1.83\left(\mathrm{~d}, J=6.9 \mathrm{~Hz}, 3 \mathrm{H}, \mathrm{CH}_{3}\right), 1.72\left(\mathrm{~m}, J=6.9,6.8 \mathrm{~Hz}, 2 \mathrm{H}, \mathrm{CH}_{2}\right), 1.62(\mathrm{~m}, J=6.9$, 
$6.8 \mathrm{~Hz}, 1 \mathrm{H}, \mathrm{CH}), 0.95\left(\mathrm{~d}, J=6.9,6 \mathrm{H}, 2 \mathrm{CH}_{3}\right) .{ }^{13} \mathrm{C} \mathrm{NMR}\left(100.61 \mathrm{MHz}, \mathrm{CDCl}_{3}\right): \delta_{\mathrm{C}} 153.6\left(\mathrm{C}^{3}\right)$, $127.4\left(\mathrm{C}^{5}\right), 102.5\left(\mathrm{C}^{4}\right), 52.3(\mathrm{CHCl}), 48.0\left(\mathrm{NCH}_{2}\right), 38.8(\mathrm{CH}), 26.01\left(\mathrm{CH}_{2}\right), 25.1\left(\mathrm{CH}_{3}\right), 22.6$ $\left(\mathrm{CH}_{3}\right)$. MS (EI, $\left.70 \mathrm{eV}\right), \mathrm{m} / \mathrm{z}(\%): 234\left(\mathrm{M}^{+}, 0,4\right), 219$ (2.5), 199 (100), 177 (98), 163 (28), 141 (30), 129 (85). Anal. Calcd for $\mathrm{C}_{10} \mathrm{H}_{16} \mathrm{Cl}_{2} \mathrm{~N}_{2}$ : C, 51.08; $\mathrm{H}$ 6.86; Cl, 30.15; N, 11.91. Found: $\mathrm{C}$, 50.07; H, 6.76; Cl, 30.31; N, 11.90.

Mixture of 3-(1-bromoethyl)-5-chloro-1-(1-heptyl)-1H-pyrazole (3g) and 5-chloro-3-(1chloroethyl)-1-(1-heptyl)-1H-pyrazole (3h). Colourless liquid $(10.2 \mathrm{~g}, \mathbf{3 g} 7.7 \mathrm{~g}, 50 \%$; 3h $2.2 \mathrm{~g}$, $18.5 \%)$, bp $142-150{ }^{\circ} \mathrm{C}(15 \mathrm{~mm} \mathrm{Hg})$, ratio $3 \mathrm{~g}$ (72\%) : $\mathbf{3 h}(28 \%)$ is determined from the intensity signals of the protons of the groups of $\mathrm{CHCl}$ and $\mathrm{CHBr}$ in the ${ }^{1} \mathrm{H}$ NMR spectrum; IR $\left(v_{\max }, \mathrm{cm}^{-1}\right)$ : 3g and 3h. 3135, $3100(=\mathrm{CH}), 2964,2979,2875\left(\mathrm{CH}_{\mathrm{Alk}}\right), 1510(\mathrm{C}=\mathrm{C}, \mathrm{C} \mathrm{N})$.

3g. ${ }^{1} \mathrm{H}$ NMR $\left(400.13 \mathrm{MHz}, \mathrm{CDCl}_{3}\right): \delta_{\mathrm{H}} 6.24\left(\mathrm{~s}, 1 \mathrm{H}, \mathrm{H}^{4}\right), 5.16(\mathrm{q}, J 6.9 \mathrm{~Hz}, 1 \mathrm{H}, \mathrm{CHBr}), 4.05(\mathrm{t}, J$ $\left.7.0 \mathrm{~Hz}, 2 \mathrm{H}, \mathrm{NCH}_{2}\right), 1.98\left(\mathrm{~d}, J 6.9 \mathrm{~Hz}, 3 \mathrm{H}, \mathrm{CH}_{3}\right), 1.80$ (dd, J 7.0, $\left.6.8 \mathrm{~Hz}, 2 \mathrm{H}, \mathrm{CH}_{2}\right), 1.27(\mathrm{~m}, 8 \mathrm{H}$, $\left.4 \mathrm{CH}_{2}\right), 0.84\left(\mathrm{t}, J 6.9 \mathrm{~Hz}, 3 \mathrm{H}, \mathrm{CH}_{3}\right)$.

3h. ${ }^{1} \mathrm{H}$ NMR (400.13 MHz, $\left.\mathrm{CDCl}_{3}\right)$ : $\delta_{\mathrm{H}} 6.24\left(\mathrm{~s}, 1 \mathrm{H}, \mathrm{H}^{4}\right), 5.05$ (q, J $\left.6.9 \mathrm{~Hz}, 1 \mathrm{H}, \mathrm{CHCl}\right), 4.05$ (t, $J$ $\left.7.0 \mathrm{~Hz}, 2 \mathrm{H}, \mathrm{NCH}_{2}\right), 1.98\left(\mathrm{~d}, J 6.9 \mathrm{~Hz}, 3 \mathrm{H}, \mathrm{CH}_{3}\right), 1.80\left(\mathrm{dd}, J 7.0,6.8 \mathrm{~Hz}, 2 \mathrm{H}, \mathrm{CH}_{2}\right), 1.27(\mathrm{~m}, 8 \mathrm{H}$, $\left.4 \mathrm{CH}_{2}\right), 0.85\left(\mathrm{t}, J 6.9 \mathrm{~Hz}, 3 \mathrm{H}, \mathrm{CH}_{3}\right)$.

5-Chloro-3-(1-chloroethyl)-1-heptyl-1H-pyrazole (3h). Colourless liquid (10.2 g, 78\%), bp 142-144 ${ }^{\circ} \mathrm{C}(15 \mathrm{~mm} \mathrm{Hg})$, IR $\left(v_{\max }, \mathrm{cm}^{-1}\right)$ : 3h: 3135, $3100(=\mathrm{CH}), 2964,2979,2875\left(\mathrm{CH}_{\text {Alk }}\right), 1510$ $(\mathrm{C}=\mathrm{C}, \mathrm{C}=\mathrm{N}) .{ }^{1} \mathrm{H} \mathrm{NMR}\left(400.13 \mathrm{MHz}, \mathrm{CDCl}_{3}\right): \delta_{\mathrm{H}} 6.24\left(\mathrm{~s}, 1 \mathrm{H}, \mathrm{H}^{4}\right), 5.05(\mathrm{q}, J=6.9 \mathrm{~Hz}, 1 \mathrm{H}, \mathrm{CHCl})$, $4.05\left(\mathrm{t}, J=7.0 \mathrm{~Hz}, 2 \mathrm{H}, \mathrm{NCH}_{2}\right), 1.98\left(\mathrm{~d}, J=6.9 \mathrm{~Hz}, 3 \mathrm{H}, \mathrm{CH}_{3}\right), 1.80\left(\mathrm{dd}, J=7.0,6.8 \mathrm{~Hz}, 2 \mathrm{H}, \mathrm{CH}_{2}\right)$, $1.27\left(\mathrm{~m}, 8 \mathrm{H}, 4 \mathrm{CH}_{2}\right), 0.85\left(\mathrm{t}, J=6.9 \mathrm{~Hz}, 3 \mathrm{H}, \mathrm{CH}_{3}\right) .{ }^{13} \mathrm{C} \mathrm{NMR}\left(100.61 \mathrm{MHz}, \mathrm{CDCl}_{3}\right): \delta_{\mathrm{C}} 153.6$ $\left(\mathrm{C}^{3}\right), 127.4\left(\mathrm{C}^{5}\right), 102.6\left(\mathrm{C}^{4}\right), 52.3(\mathrm{CHCl}), 48.0\left(\mathrm{NCH}_{2}\right), 38,75(\mathrm{CH}), 26.0\left(\mathrm{CH}_{2}\right), 25.1\left(\mathrm{CH}_{3}\right)$, $22.6\left(\mathrm{CH}_{3}\right)$.

Anal. Calcd for $\mathrm{Cl}_{12} \mathrm{H}_{20} \mathrm{Cl}_{2} \mathrm{~N}_{2}$ : C, 54.76; H 7.66; Cl, 26.94; N, 10.64. Found: C, 54.46; H, 7.75; $\mathrm{Cl}, 26.81 ; \mathrm{N}, 10.80$.

\section{General procedure for the synthesis of pyrazole derivatives $3 \mathbf{i}, \mathbf{j}$}

Dimethylhydrazine (100 mmol, $6.1 \mathrm{~g}$ ) was added dropwise to a solution of dichlorovinylketone 2c,d $(50 \mathrm{mmol}, 12.3 \mathrm{~g})^{28}$ in diethyl ether $(150 \mathrm{~mL})$ for $20 \mathrm{~min}$. Then the reaction mass was treated as described for $\mathbf{3 c - h}$ and $\mathbf{4 c - e}$.

3-(1-Bromo-1-methylethyl)-5-chloro-1-methyl-1H-pyrazole (3i). Obtained in mixture with pyrazole $\mathbf{4 f}(80 \% \mathbf{3 i}$ and $20 \% \mathbf{4 f})$. The mixture was used for further synthesis without separation. Light yellow liquid (10.1 g 92\%), (8.7 g, 3i, $1.4 \mathrm{~g}, \mathbf{4 f})$.

3i. ${ }^{1} \mathrm{H}$ NMR (400.13 MHz, $\left.\mathrm{CDCl}_{3}\right)$ : $\delta_{\mathrm{H}} 6.41\left(\mathrm{~s}, 1 \mathrm{H}, \mathrm{H}^{4}\right), 3.82\left(\mathrm{~s}, 3 \mathrm{H}, \mathrm{NCH}_{3}\right), 1.95\left(\mathrm{~s}, 6 \mathrm{H}, \mathrm{CH}_{3}\right)$.

${ }^{13} \mathrm{C}$ NMR $\left(100.61 \mathrm{MHz}, \mathrm{CDCl}_{3}\right): \delta_{\mathrm{C}} 156.6\left(\mathrm{C}^{3}\right), 135.4\left(\mathrm{C}^{5}\right), 128.1(\mathrm{C}=), 113.6\left(\mathrm{CH}_{2}\right), 101.7\left(\mathrm{C}^{4}\right)$, $32.6\left(\mathrm{NCH}_{3}\right), 19.8\left(\mathrm{CH}_{3}\right)$.MS (EI, $\left.70 \mathrm{eV}\right), \mathrm{m} / \mathrm{z}(\%): 236\left(\mathrm{M}^{+}, 9\right), 157(55), 143(100)$.

4f. ${ }^{1} \mathrm{H}$ NMR (400.13 MHz, $\left.\mathrm{CDCl}_{3}\right): \delta_{\mathrm{H}} 6.31\left(\mathrm{~s}, 1 \mathrm{H}, \mathrm{H}^{4}\right), 5.44(\mathrm{~s}, 1 \mathrm{H},=\mathrm{CH}), 5.06(\mathrm{~s}, 1 \mathrm{H},=\mathrm{CH})$, $3.82\left(\mathrm{~s}, 3 \mathrm{H}, \mathrm{NCH}_{3}\right), 2.09\left(\mathrm{~s}, 3 \mathrm{H}, \mathrm{CH}_{3}\right) .{ }^{13} \mathrm{C} \mathrm{NMR}\left(100.61 \mathrm{MHz}, \mathrm{CDCl}_{3}\right): \delta_{\mathrm{C}} 152.1\left(\mathrm{C}^{3}\right), 136.4$ $\left(\mathrm{C}^{5}\right), 127.7\left(\mathrm{C}^{4}\right), 112.2(=\mathrm{CH}), 101.4(=\mathrm{CH}), 36.1\left(\mathrm{NCH}_{3}\right), 19.7\left(\mathrm{CH}_{3}\right)$. 
3-(1-Bromopropyl)-5-chloro-1-methyl-1 $\boldsymbol{H}$-pyrazole (3j). Light yellow liquid, (9.3 g, 78\%). ${ }^{1} \mathrm{H}$ NMR (400.13 MHz, $\left.\mathrm{CDCl}_{3}\right): \delta_{\mathrm{H}} 6.26\left(\mathrm{~s}, 1 \mathrm{H}, \mathrm{H}^{4}\right), 4.90(\mathrm{t}, J=7.4 \mathrm{~Hz}, 1 \mathrm{H}, \mathrm{CHBr}), 3.81$ (s, 3H, $\left.\mathrm{NCH}_{3}\right), 2.20\left(\mathrm{~m}, J=7.4,7.2 \mathrm{~Hz}, 2 \mathrm{H}, \mathrm{CH}_{2}\right), 1.04\left(\mathrm{t}, J=7.2 \mathrm{~Hz}, 3 \mathrm{H}, \mathrm{CH}_{3}\right) .{ }^{13} \mathrm{C}$ NMR $(100.61 \mathrm{MHz}$, $\left.\mathrm{CDCl}_{3}\right): \delta_{\mathrm{C}} 152.9\left(\mathrm{C}^{3}\right), 128.0\left(\mathrm{C}^{5}\right), 102.9\left(\mathrm{C}^{4}\right), 49.2(\mathrm{CHBr}), 36.2\left(\mathrm{NCH}_{3}\right), 32.0\left(\mathrm{CH}_{2}\right), 12.7$ $\left(\mathrm{CH}_{3}\right)$. MS (EI, $\left.70 \mathrm{eV}\right), \mathrm{m} / \mathrm{z}(\%): 236\left(\mathrm{M}^{+}, 0.4\right), 207$ (1), 157 (100), 128 (38). Anal. Calcd for $\mathrm{C}_{7} \mathrm{H}_{10} \mathrm{BrClN}_{2}$ : C, 35.40; H 4.24; Br, 33.64; $\mathrm{Cl}, 14.93 ; \mathrm{N}, 11.79$. Found: C, 35.28; H, 4.38; $\mathrm{Br}$, 33.44; $\mathrm{Cl}, 14.68 ; \mathrm{N}, 11.64$.

5-Chloro-3-ethenyl-1-(1-methylethyl)-1H-pyrazole (4c). Obtained from mixture of 3c and 3d or 3d, the reaction time was $20 \mathrm{~min}$. Colourless liquid $(5.16 \mathrm{~g}, 61 \%)$, bp 56-58 ${ }^{\circ} \mathrm{C}(1 \mathrm{~mm} \mathrm{Hg})$. IR $\left(v_{\max }, \mathrm{cm}^{-1}\right): 3137,3018,3091(=\mathrm{CH}), 2944,2852\left(\mathrm{CH}_{\mathrm{Alk}}\right), 1638,1505(\mathrm{C}=\mathrm{C}, \mathrm{C}=\mathrm{N}) .{ }^{1} \mathrm{H}$ NMR $\left(400.13 \mathrm{MHz}, \mathrm{CDCl}_{3}\right): \delta_{\mathrm{H}} 6.65(\mathrm{dd}, J 11.0,17.1 \mathrm{~Hz}, 1 \mathrm{H}, \mathrm{CH}=), 6.30\left(\mathrm{~s}, 1 \mathrm{H}, \mathrm{H}^{4}\right), 5.65(\mathrm{dd}, J 1.1$, $\left.17.1 \mathrm{~Hz}, 1 \mathrm{H},=\mathrm{CH}_{2}\right), 5.27\left(\mathrm{dd}, J 1.1,11.0,1 \mathrm{H},=\mathrm{CH}_{2}\right), 4.62(\mathrm{spt}, J 6.7 \mathrm{~Hz}, 1 \mathrm{H}, \mathrm{CH}), 1.45(\mathrm{~d}, J 6.7$ $\left.\mathrm{Hz}, 6 \mathrm{H}, 2 \mathrm{CH}_{3}\right) .{ }^{13} \mathrm{C}$ NMR $\left(100.61 \mathrm{MHz}, \mathrm{CDCl}_{3}\right): \delta_{\mathrm{C}} 150.3\left(\mathrm{C}^{3}\right), 129.3(\mathrm{CH}=), 126.5\left(\mathrm{C}^{5}\right), 115.4$

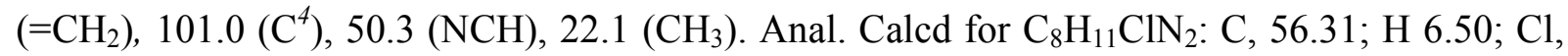
20.78; N, 16.42. Found: C, 56.37; H, 6.58; Cl, 20.53; N, $16.39 \%$.

5-Chloro-3-ethenyl-1-(3-methylbutyl)-1H-pyrazole (4d). Obtained from mixture of $\mathbf{3 e}$ and $\mathbf{3 f}$ or 3f, the reaction time was $20 \mathrm{~min}$. Colourless liquid $(8.4 \mathrm{~g}, 85 \%)$, bp $79-82{ }^{\circ} \mathrm{C}(15 \mathrm{~mm} \mathrm{Hg}) .{ }^{1} \mathrm{H}$ NMR (400.13 MHz, $\left.\mathrm{CDCl}_{3}\right): \delta_{\mathrm{H}} 6.61(\mathrm{dd}, J 11.1,17.7 \mathrm{~Hz}, 1 \mathrm{H}, \mathrm{CH}=), 6.29\left(\mathrm{~s}, 1 \mathrm{H}, \mathrm{H}^{4}\right), 5.62(\mathrm{dd}$, $\left.J 1.2,17.7 \mathrm{~Hz}, 1 \mathrm{H},=\mathrm{CH}_{2}\right), 5.26\left(\mathrm{dd}, J 1.2,11.1 \mathrm{~Hz}, 1 \mathrm{H},=\mathrm{CH}_{2}\right), 4.08\left(\mathrm{t}, J 7.5 \mathrm{~Hz}, 2 \mathrm{H}, \mathrm{NCH}_{2}\right)$, $1.71\left(\mathrm{~m}, J 7.5,6.8 \mathrm{~Hz}, 2 \mathrm{H}, \mathrm{CH}_{2}\right), 1.62(\mathrm{~m}, J 6.8,6.5 \mathrm{~Hz}, 1 \mathrm{H}, \mathrm{CH}), 0.94\left(\mathrm{~d}, J 6.5 \mathrm{~Hz}, 6 \mathrm{H}, 2 \mathrm{CH}_{3}\right)$. ${ }^{13} \mathrm{C} \mathrm{NMR}\left(100.61 \mathrm{MHz}, \mathrm{CDCl}_{3}\right): \delta_{\mathrm{C}} 150.5\left(\mathrm{C}^{3}\right), 129.2(\mathrm{CH}=), 127.4\left(\mathrm{C}^{5}\right), 115.6\left(=\mathrm{CH}_{2}\right), 101.3$ $\left(\mathrm{C}^{4}\right), 47.8\left(\mathrm{NCH}_{2}\right), 38.8(\mathrm{CH}), 25.9\left(\mathrm{CH}_{2}\right), 22.5\left(\mathrm{CH}_{3}\right) \mathrm{ppm}$. Anal. Calcd for $\mathrm{C}_{10} \mathrm{H}_{15} \mathrm{ClN}_{2}: \mathrm{C}$, 60.45; H 7.61; Cl, 17.84; N, 14.10. Found: C, 60.37; H, 7.58; Cl, 17.53; N, $13.89 \%$.

5-Chloro-3-ethenyl)-1-heptyl-1H-pyrazole (4e). Obtained from mixture of $\mathbf{3 g}$ and $\mathbf{3 h}$, the reaction time was $10 \mathrm{~min}$. Colourless liquid $(7.17 \mathrm{~g}, 63 \%)$, bp $165-169^{\circ} \mathrm{C}(25 \mathrm{~mm} \mathrm{Hg}) . \mathrm{IR}\left(v_{\max }\right.$, $\left.\mathrm{cm}^{-1}\right)$ : 3137, $3100(=\mathrm{CH}), 2964,2957,2857\left(\mathrm{CH}_{\mathrm{Alk}}\right), 1504(\mathrm{C}=\mathrm{C}, \mathrm{C}=\mathrm{N}) .{ }^{1} \mathrm{H}$ NMR $(400.13 \mathrm{MHz}$, $\left.\mathrm{CDCl}_{3}\right): \delta_{\mathrm{H}} 6.58(\mathrm{dd}, J 11.0,17.7 \mathrm{~Hz}, 1 \mathrm{H}, \mathrm{CH}=), 6.25\left(\mathrm{~s}, 1 \mathrm{H}, \mathrm{H}^{4}\right), 5.62(\mathrm{dd}, J 2.7,17.7 \mathrm{~Hz}, 1 \mathrm{H}$, $\left.=\mathrm{CH}_{2}\right), 5.24\left(\mathrm{dd}, J 2.7,11.0 \mathrm{~Hz}, 1 \mathrm{H},=\mathrm{CH}_{2}\right), 4.02\left(\mathrm{t}, J 7.4 \mathrm{~Hz}, 2 \mathrm{H}, \mathrm{NCH}_{2}\right), 1.77\left(\mathrm{~m}, 2 \mathrm{H}, \mathrm{CH}_{2}\right)$, $1.25\left(\mathrm{~m}, 8 \mathrm{H}, 4 \mathrm{CH}_{2}\right), 0.82\left(\mathrm{t}, J 6.9 \mathrm{~Hz}, 3 \mathrm{H}, \mathrm{CH}_{3}\right) .{ }^{13} \mathrm{C} \mathrm{NMR}\left(100.61 \mathrm{MHz}, \mathrm{CDCl}_{3}\right): \delta_{\mathrm{C}} 150.4\left(\mathrm{C}^{3}\right)$, $129.1(\mathrm{CH}=), 127.4\left(\mathrm{C}^{5}\right), 115.5\left(=\mathrm{CH}_{2}\right), 101.2\left(\mathrm{C}^{4}\right), 49.2\left(\mathrm{NCH}_{2}\right), 31.7\left(\mathrm{CH}_{2}\right), 30.0\left(\mathrm{CH}_{2}\right), 28.9$ $\left(\mathrm{CH}_{2}\right), 26.5\left(\mathrm{CH}_{2}\right), 22.6\left(\mathrm{CH}_{2}\right), 14.1\left(\mathrm{CH}_{3}\right)$. Anal. Calcd for $\mathrm{C}_{12} \mathrm{H}_{19} \mathrm{ClN}_{2}: \mathrm{C}, 63.56 ; \mathrm{H}, 8.45 ; \mathrm{Cl}$, 15.64; N, 12.35. Found: C, 63.69; H, 8.57; Cl, 15.25; N, $12.57 \%$.

5-Chloro-1-methyl-3-(1-methylethenyl)-1 $\mathbf{H}$-pyrazole (4f). Obtained from mixture of $\mathbf{3 i}$ and $\mathbf{4 f}$ The reaction time was $20 \mathrm{~min}$. Colourless liquid $(5.33 \mathrm{~g}, 68 \%)$, bp $56{ }^{\circ} \mathrm{C}(1 \mathrm{~mm} \mathrm{Hg}) . \mathrm{IR}\left(v_{\max }\right.$, $\left.\mathrm{cm}^{-1}\right)$ : 3137, $3100(=\mathrm{CH}), 2964,2957,2857\left(\mathrm{CH}_{\mathrm{Alk}}\right), 1504(\mathrm{C}=\mathrm{C}, \mathrm{C}=\mathrm{N}) .{ }^{1} \mathrm{H}$ NMR $(400.13 \mathrm{MHz}$, $\left.\mathrm{CDCl}_{3}\right): \delta_{\mathrm{H}} 6.31\left(\mathrm{~s}, 1 \mathrm{H}, \mathrm{H}^{4}\right), 5.44(\mathrm{~s}, 1 \mathrm{H},=\mathrm{CH}), 5.06(\mathrm{~s}, 1 \mathrm{H},=\mathrm{CH}), 3.82\left(\mathrm{~s}, 3 \mathrm{H}, \mathrm{CH}_{3}\right), 2.09(\mathrm{~s}$, $\left.3 \mathrm{H}, \mathrm{CH}_{3}\right) .{ }^{13} \mathrm{C} \mathrm{NMR}\left(100.61 \mathrm{MHz}, \mathrm{CDCl}_{3}\right): \delta_{\mathrm{C}} 152.1\left(\mathrm{C}^{3}\right), 136.4\left(\mathrm{C}^{5}\right), 127.7\left(\mathrm{C}^{4}\right), 112.2(=\mathrm{CH})$, $101.4(=\mathrm{CH}), 36.1\left(\mathrm{NCH}_{3}\right), 19.7\left(\mathrm{CH}_{3}\right)$. Anal. Calcd for $\mathrm{C}_{7} \mathrm{H}_{9} \mathrm{ClN}_{2}: \mathrm{C}, 53.68 ; \mathrm{H}, 5.79 ; \mathrm{Cl}, 22.64$; $\mathrm{N}, 17.89$. Found: C, 53.33; H, 5.62; Cl, 22.28; N, $18.04 \%$. 
5-Chloro-1-methyl-3-[prop-1-en-1-yl]-1H-pyrazole (4g). The reaction time was 20 min. Colourless liquid (6.42 g, 82\% from pyrazole 3j), (mixture of $E$ and $Z$-isomers). The ratio $\mathrm{E} / \mathrm{Z}$ isomer $4: 1$; bp $86{ }^{\circ} \mathrm{C}(2 \mathrm{~mm} \mathrm{Hg}) .{ }^{1} \mathrm{H}$ NMR $\left(400.13 \mathrm{MHz}, \mathrm{CDCl}_{3}\right): \delta_{\mathrm{H}} 6.31-6.14(\mathrm{~m}, 3 \mathrm{H}$, $\left.\mathrm{CH}=\mathrm{CH}, \mathrm{H}^{4}\right), 3.82,3.78\left(\mathrm{~s}, 3 \mathrm{H}, \mathrm{NCH}_{3}\right), 1.96,1.86\left(\mathrm{~d}, J=6.4 \mathrm{~Hz}, 3 \mathrm{H}, \mathrm{CH}_{3}\right) .{ }^{13} \mathrm{C} \mathrm{NMR}(100.61$ $\left.\mathrm{MHz}, \mathrm{CDCl}_{3}\right): \delta_{\mathrm{C}} 150.3\left(\mathrm{C}^{3}\right), 127.9\left(\mathrm{C}^{5}\right), 122.8\left(\mathrm{C}^{4}\right), 109.6(=\mathrm{CH}), 104.8(=\mathrm{CH}), 100.9(=\mathrm{CH})$, 37.3, $35.9\left(\mathrm{NCH}_{3}\right), 18.2\left(\mathrm{CH}_{3}\right)$. Anal. Calcd for $\mathrm{C}_{7} \mathrm{H}_{9} \mathrm{ClN}_{2}: \mathrm{C}, 53.68 ; \mathrm{H}, 5.79 ; \mathrm{Cl}, 22.64 ; \mathrm{N}$, 17.89. Found: C, 53.63; H, 5.82; Cl, 22.48; N, 17.65 .

5-Chloro-1-methyl-3-(1-methylethenyl)-1H-pyrazole (4f). Obtained from mixture of $3 \mathbf{i}$ and $\mathbf{4 f}$. The reaction time was $20 \mathrm{~min}$. Colourless liquid $(5.33 \mathrm{~g}, 68 \%)$, bp $56{ }^{\circ} \mathrm{C}(1 \mathrm{~mm} \mathrm{Hg}) . \mathrm{IR}\left(v_{\max }\right.$, $\left.\mathrm{cm}^{-1}\right)$ : 3137, $3100(=\mathrm{CH}), 2964,2957,2857\left(\mathrm{CH}_{\mathrm{Alk}}\right), 1504(\mathrm{C}=\mathrm{C}, \mathrm{C}=\mathrm{N}) .{ }^{1} \mathrm{H}$ NMR $(400.13 \mathrm{MHz}$, $\left.\mathrm{CDCl}_{3}\right): \delta_{\mathrm{H}} 6.31\left(\mathrm{~s}, 1 \mathrm{H}, \mathrm{H}^{4}\right), 5.44\left(\mathrm{~s}, 1 \mathrm{H},=\mathrm{CH}_{2}\right), 5.06\left(\mathrm{~s}, 1 \mathrm{H},=\mathrm{CH}_{2}\right), 3.82\left(\mathrm{~s}, 3 \mathrm{H}, \mathrm{CH}_{3}\right), 2.08(\mathrm{~s}$, $\left.3 \mathrm{H}, \mathrm{CH}_{3}\right) .{ }^{13} \mathrm{C} \mathrm{NMR}\left(100.61 \mathrm{MHz}, \mathrm{CDCl}_{3}\right): \delta_{\mathrm{C}} 152.1\left(\mathrm{C}^{3}\right), 136.4\left(\mathrm{C}^{5}\right), 127.7\left(\mathrm{C}^{4}\right), 112.2(=\mathrm{CH})$, $101.4(=\mathrm{CH}), 36.1\left(\mathrm{NCH}_{3}\right), 19.7\left(\mathrm{CH}_{3}\right)$. Anal. Calcd for $\mathrm{C}_{7} \mathrm{H}_{9} \mathrm{ClN}_{2}: \mathrm{C}, 53.68 ; \mathrm{H}, 5.79 ; \mathrm{Cl}, 22.64$; N, 17.89. Found: C, 53.33; H, 5.62; Cl, 22.28; N, $18.04 \%$.

5-Chloro-1-methyl-3-[prop-1-en-1-yl]-1H-pyrazole (4g). The reaction time was $20 \mathrm{~min}$. Colourless liquid ( $6.42 \mathrm{~g}$, $82 \%$ yield, from pyrazole $3 \mathbf{j}$, mixture of $E$ and $Z$-isomers, the ratio $\mathrm{E} / \mathrm{Z}$ isomer 4:1), bp $86{ }^{\circ} \mathrm{C}(2 \mathrm{~mm} \mathrm{Hg}) .{ }^{1} \mathrm{H}$ NMR (400.13 MHz, $\left.\mathrm{CDCl}_{3}\right): \delta_{\mathrm{H}} 6.31-6.14(\mathrm{~m}, 3 \mathrm{H}$, $\left.\mathrm{CH}=\mathrm{CH}, \mathrm{H}^{4}\right), 3.82,3.78\left(\mathrm{~s}, 3 \mathrm{H}, \mathrm{NCH}_{3}\right), 1.96,1.86\left(\mathrm{~d}, J=6.4 \mathrm{~Hz}, 3 \mathrm{H}, \mathrm{CH}_{3}\right) .{ }^{13} \mathrm{C}_{\mathrm{NMR}}(100.61$ $\left.\mathrm{MHz}, \mathrm{CDCl}_{3}\right): \delta_{\mathrm{C}} 148.5\left(\mathrm{C}^{3}\right), 127.5\left(\mathrm{C}^{5}\right), 126.8\left(\mathrm{C}^{4}\right), 107.7(=\mathrm{CH}), 103.94(=\mathrm{CH}), 36.9,35.6$ $\left(\mathrm{NCH}_{3}\right), 14.8,14.38\left(\mathrm{CH}_{3}\right)$. Anal. Calcd for $\mathrm{C}_{7} \mathrm{H}_{9} \mathrm{ClN}_{2}$ : C, 53.68; H, 5.79; $\mathrm{Cl}, 22.64 ; \mathrm{N}, 17.89$. Found: C, 53.63; H, 5.82; Cl, 22.48; N, $17.65 \%$.

General procedure for the synthesis of 1-alkyl-3-[2-(arylsulfanyl)alkyl]-1H-pyrazoles 6.

3-Alkenyl-5-chloropyrazole 4 ( $1 \mathrm{mmol})$ and aromatic thiol 5 (1 mmol) were mixed and kept for 1-3 min. The resulting pyrazoles 6 were washed with hexane and dried under vacuum.

5-Chloro-1-methyl-3-[2-(phenylsulfanyl)ethyl]-1 $\mathrm{H}$-pyrazole (6aa). Obtained from vinylpyrazole $4 \mathbf{a}(0.14 \mathrm{~g})$ and benzenethiol $5 \mathbf{a}(0.11 \mathrm{~g})$. Colourless oil, bp $210-212{ }^{\circ} \mathrm{C}(15 \mathrm{~mm} \mathrm{Hg})$, 0.22 g, 86\% yield. ${ }^{1} \mathrm{H}$ NMR $\left(400.13 \mathrm{MHz}, \mathrm{CDCl}_{3}\right): \delta_{\mathrm{H}} 7.49-7.14\left(\mathrm{~m}, 5 \mathrm{H}, \mathrm{C}_{6} \mathrm{H}_{5}\right), 6.03(\mathrm{~s}, 1 \mathrm{H}$, $\mathrm{H}_{P y r}^{4}$ ), $3.75\left(\mathrm{~s}, 3 \mathrm{H}, \mathrm{NCH}_{3}\right), 3.17\left(\mathrm{t}, J 7.4 \mathrm{~Hz}, 2 \mathrm{H}, \mathrm{SCH}_{2}\right), 2.87\left(\mathrm{t}, J 7.4 \mathrm{~Hz}, 2 \mathrm{H}, \mathrm{CH}_{2} \mathrm{CH}_{2} \mathrm{~S}\right) .{ }^{13} \mathrm{C}$ NMR (100.61 MHz, $\left.\mathrm{CDCl}_{3}\right): \delta_{\mathrm{C}} 150.5\left(\mathrm{C}_{\text {Pyr }}^{3}\right), 136.2\left(\mathrm{C}^{i}\right), 129.1\left(\mathrm{C}^{2,6}\right), 128.9\left(\mathrm{C}^{3,5}\right), 127.2\left(\mathrm{C}_{\text {Pyr }}^{5}\right)$, $126.1\left(\mathrm{C}^{4}\right), 103.6\left(\mathrm{C}_{P y r}^{4}\right), 35.9\left(\mathrm{NCH}_{3}\right), 33.2\left(\mathrm{SCH}_{2}\right), 28.7\left(\mathrm{CH}_{2} \mathrm{CH}_{2} \mathrm{~S}\right)$. Anal. Calcd for $\mathrm{C}_{12} \mathrm{H}_{13} \mathrm{ClN}_{2} \mathrm{~S}$ : C, 57.02; H, 5.18; Cl, 14.03; N, 11.08; S 12.68. Found: C, 57.06; H, 5.19; Cl, $14.05 ; \mathrm{N}, 11.04 ; \mathrm{S}, 12.65 \%$.

5-Chloro-3-\{2-[(4-chlorophenyl)sulfanyl]ethyl\}-1-methyl-1H-pyrazole (6ab). Obtained from vinylpyrazole $4 \mathbf{a}(0.14 \mathrm{~g})$ and 4-chlorobenzenethiol $5 \mathbf{b}(0.14 \mathrm{~g})$. Colourless oil $(0.26 \mathrm{~g}, 94 \%)$, bp 243-246 ${ }^{\circ} \mathrm{C}(15 \mathrm{~mm} \mathrm{Hg}) .{ }^{1} \mathrm{H}$ NMR $\left(400.13 \mathrm{MHz}, \mathrm{CDCl}_{3}\right): \delta_{\mathrm{H}} 7.26,7.20\left(\mathrm{AA}^{\prime} \mathrm{BB}^{\prime}, J 6.6 \mathrm{~Hz}, 4 \mathrm{H}\right.$, $\left.\mathrm{C}_{6} \mathrm{H}_{4}\right), 6.00\left(\mathrm{~s}, 1 \mathrm{H}, \mathrm{H}_{P y r}^{4}\right), 3.74\left(\mathrm{~s}, 3 \mathrm{H}, \mathrm{NCH}_{3}\right), 3.12\left(\mathrm{t}, J 7.5 \mathrm{~Hz}, 2 \mathrm{H}, \mathrm{SCH}_{2}\right), 2.83(\mathrm{t}, J 7.5 \mathrm{~Hz}$, $\left.2 \mathrm{H}, \mathrm{CH}_{2} \mathrm{CH}_{2} \mathrm{~S}\right) .{ }^{13} \mathrm{C} \mathrm{NMR}\left(100.61 \mathrm{MHz}, \mathrm{CDCl}_{3}\right): \delta_{\mathrm{C}} 150.4\left(\mathrm{C}_{P y r}^{3}\right), 134.8\left(\mathrm{C}^{i}\right), 132.2\left(\mathrm{C}^{4}\right), 131.0$ $\left(\mathrm{C}^{3,5}\right), 129.1\left(\mathrm{C}^{2,6}\right), 127.5\left(\mathrm{C}_{P y r}^{5}\right), 103.7\left(\mathrm{C}_{P y r}^{4}\right), 36.1\left(\mathrm{NCH}_{3}\right), 33.6\left(\mathrm{SCH}_{2}\right), 28.8\left(\underline{\mathrm{CH}}_{2} \mathrm{CH}_{2} \mathrm{~S}\right)$. Anal. Calcd for $\mathrm{C}_{12} \mathrm{H}_{12} \mathrm{Cl}_{2} \mathrm{~N}_{2} \mathrm{~S}$ : C, 50.18; H, 4.21; Cl, 24.69; N, 9.75; S, 11.16. Found: C, 50.22; $\mathrm{H}, 4.20 ; \mathrm{Cl}, 24.65 ; \mathrm{N}, 9.76 ; \mathrm{S}, 11.18 \%$. 
3-\{2-[(4-Bromophenyl)sulfanyl]ethyl\}-5-chloro-1-methyl-1H-pyrazole (6ac). Obtained from vinylpyrazole $4 \mathbf{a}(0.14 \mathrm{~g})$ and 4-bromobenzenethiol 5c $(0.19 \mathrm{~g})$. Colourless oil $(0.30 \mathrm{~g}, 92 \%)$, bp 259-264 ${ }^{\circ} \mathrm{C}(15 \mathrm{~mm} \mathrm{Hg}) .{ }^{1} \mathrm{H}$ NMR $\left(400.13 \mathrm{MHz}, \mathrm{CDCl}_{3}\right): \delta_{\mathrm{H}} 7.32,7.14\left(\mathrm{AA}^{\prime} \mathrm{BB}^{\prime}, J 8.7 \mathrm{~Hz}, 4 \mathrm{H}\right.$, $\left.\mathrm{C}_{6} \mathrm{H}_{4}\right), 5.96\left(\mathrm{~s}, 1 \mathrm{H}, \mathrm{H}_{P y r}^{4}\right), 3.69\left(\mathrm{~s}, 3 \mathrm{H}, \mathrm{NCH}_{3}\right), 3.09\left(\mathrm{t}, J 7.3 \mathrm{~Hz}, 2 \mathrm{H}, \mathrm{SCH}_{2}\right), 2.80$ (t, J 7.3 Hz, $\left.2 \mathrm{H}, \mathrm{CH}_{2} \mathrm{CH}_{2} \mathrm{~S}\right) .{ }^{13} \mathrm{C} \mathrm{NMR}\left(100.61 \mathrm{MHz}, \mathrm{CDCl}_{3}\right): \delta_{\mathrm{C}} 150.2\left(\mathrm{C}_{P y r}^{3}\right), 135.5\left(\mathrm{C}^{i}\right), 131.9\left(\mathrm{C}^{2}\right), 131.0$ $\left(\mathrm{C}^{3}\right), 127.3\left(\mathrm{C}_{P y r}^{5}\right), 119.9\left(\mathrm{C}^{4}\right), 103.6\left(\mathrm{C}_{P y r}^{4}\right), 36.0\left(\mathrm{NCH}_{3}\right), 33.3\left(\mathrm{SCH}_{2}\right), 28.6\left(\mathrm{CH}_{2} \mathrm{CH}_{2} \mathrm{~S}\right)$. Anal. Calcd for $\mathrm{C}_{12} \mathrm{H}_{12} \mathrm{BrClN}_{2} \mathrm{~S}$ : C, 43.46; H, 3.65; N, 8.45; S, 9.67. Found: C, 43.49; H, 3.65; N, 8.46; $\mathrm{S}, 9.64 \%$.

5-Chloro-1-methyl-3-\{2-[(4-methylphenyl)sulfanyl]ethyl\}-1 $\boldsymbol{H}$-pyrazole (6ad). Obtained from vinylpyrazole $4 \mathbf{a}(0.14 \mathrm{~g})$ and 4-methylbenzenethiol 5d (0.12 g). Colourless oil $(0.23 \mathrm{~g}, 90 \%)$, bp 230-234 ${ }^{\circ} \mathrm{C}(15 \mathrm{~mm} \mathrm{Hg}) .{ }^{1} \mathrm{H}$ NMR $\left(400.13 \mathrm{MHz}, \mathrm{CDCl}_{3}\right): \delta_{\mathrm{H}} 7.24,7.06,\left(\mathrm{AA}^{\prime} \mathrm{BB}^{\prime}, J 8.2 \mathrm{~Hz}, 4 \mathrm{H}\right.$, $\left.\mathrm{C}_{6} \mathrm{H}_{4}\right), 6.00\left(\mathrm{~s}, 1 \mathrm{H}, \mathrm{H}_{P y r}^{4}\right), 3.72\left(\mathrm{~s}, \mathrm{H}, \mathrm{NCH}_{3}\right), 3.09\left(\mathrm{t}, J 7.3 \mathrm{~Hz}, 2 \mathrm{H}, \mathrm{SCH}_{2}\right), 2.82(\mathrm{t}, J 7.3 \mathrm{~Hz}, 2 \mathrm{H}$, $\left.\mathrm{C}_{2} \mathrm{CH}_{2} \mathrm{~S}\right), 2.28\left(\mathrm{~s}, 3 \mathrm{H}, \mathrm{CH}_{3}\right) .{ }^{13} \mathrm{C} \mathrm{NMR}\left(100.61 \mathrm{MHz}, \mathrm{CDCl}_{3}\right): \delta_{\mathrm{C}} 150.7\left(\mathrm{C}_{\text {Pyr }}^{3}\right), 136.4\left(\mathrm{C}^{4}\right)$, $132.3\left(\mathrm{C}^{i}\right), 130.6\left(\mathrm{C}^{2,6}\right), 129.8\left(\mathrm{C}^{3,5}\right), 127.4\left(\mathrm{C}_{P y r}^{5}\right), 103.7\left(\mathrm{C}^{4}{ }_{P y r}\right), 36.0\left(\mathrm{NCH}_{3}\right), 34.1\left(\mathrm{SCH}_{2}\right), 28.9$ $\left.\mathrm{CH}_{2} \mathrm{CH}_{2} \mathrm{~S}\right), 20.9\left(\mathrm{CH}_{3}\right)$. Anal. Calcd for $\mathrm{C}_{13} \mathrm{H}_{15} \mathrm{ClN}_{2} \mathrm{~S}: \mathrm{C}, 58.53 ; \mathrm{H}, 5.67 ; \mathrm{Cl}, 13.29 ; \mathrm{N}, 10.50 ; \mathrm{S}$, 12.02. Found: $\mathrm{C}, 58.58 ; \mathrm{H}, 5.66 ; \mathrm{Cl}, 13.33 ; \mathrm{N}, 10.51 ; \mathrm{S} 12.00 \%$.

1-Benzyl-5-chloro-3-[2-(phenylsulfanyl)ethyl]-1H-pyrazole (6ba). Obtained from vinylpyrazole $4 \mathbf{b}(0.22 \mathrm{~g})$ and benzenethiol 5a $(0.11 \mathrm{~g})$. Colourless oil $(0.29 \mathrm{~g}, 89 \%)$, bp 266-270 ${ }^{\circ} \mathrm{C}$ $(15 \mathrm{~mm} \mathrm{Hg}) .{ }^{1} \mathrm{H}$ NMR $\left(400.13 \mathrm{MHz}, \mathrm{CDCl}_{3}\right): \delta_{\mathrm{H}} 7.86-7.65\left(\mathrm{~m}, 10 \mathrm{H}, \mathrm{C}_{6} \mathrm{H}_{5} \mathrm{~S}, \mathrm{C}_{6} \mathrm{H}_{5}\right), 6.59(\mathrm{~s}, 1 \mathrm{H}$, $\mathrm{H}_{\text {Pyr }}^{4}$ ), 5.77 (s, 2H, $\left.\mathrm{NCH}_{2}\right), 3.69\left(\mathrm{t}, J 7.3 \mathrm{~Hz}, 2 \mathrm{H}, \mathrm{SCH}_{2}\right), 3.42\left(\mathrm{t}, J 7.3 \mathrm{~Hz}, 2 \mathrm{H}, \mathrm{CH}_{2} \mathrm{CH}_{2} \mathrm{~S}\right) .{ }^{13} \mathrm{C}$ NMR $\left(100.61 \mathrm{MHz}, \mathrm{CDCl}_{3}\right): \delta_{\mathrm{C}} 151.1\left(\mathrm{C}_{P y r}^{3}\right), 136.1\left(\mathrm{C}_{S}^{i}\right), 129.6\left(\mathrm{C}_{S}^{2}\right), 129.1\left(\mathrm{C}_{B n}^{i}\right), 129.0\left(\mathrm{C}_{S}^{3}\right)$, $128.8\left(\mathrm{C}_{B n}^{3}\right), 127.9\left(\mathrm{C}_{B n}^{4}\right), 127.9\left(\mathrm{C}_{P y r}^{5}\right), 127.3\left(\mathrm{C}_{B n}^{2}\right), 126.2\left(\mathrm{C}_{S}^{4}\right), 104.2\left(\mathrm{C}^{4}{ }_{\text {Pyr }}\right), 52.6\left(\mathrm{NCH}_{2}\right)$, $33.3\left(\mathrm{SCH}_{2}\right), 28.8\left(\mathrm{CH}_{2} \mathrm{CH}_{2} \mathrm{~S}\right)$. Anal. Calcd for $\mathrm{C}_{18} \mathrm{H}_{17} \mathrm{ClN}_{2} \mathrm{~S}: \mathrm{C}, 65.74 ; \mathrm{H}, 5.21 ; \mathrm{Cl}, 10.78 ; \mathrm{N}$, 8.52; S, 9.75. Found: C, 65.79; H, 5.22; Cl, 10.75; N, 8.50; S, $9.77 \%$.

1-Benzyl-5-chloro-3-\{2-[(4-chlorophenyl)sulfanyl]ethyl\}-1H-pyrazole (6bb). Obtained from vinylpyrazole $4 \mathbf{b}(0.22 \mathrm{~g})$ and 4-chlorobenzenethiol $\mathbf{5 b}(0.14 \mathrm{~g})$. Colourless oil (0.34 g, 94\%), bp 275-279 ${ }^{\circ} \mathrm{C}(5 \mathrm{~mm} \mathrm{Hg}) .{ }^{1} \mathrm{H}$ NMR $\left(400.13 \mathrm{MHz}, \mathrm{CDCl}_{3}\right): \delta_{\mathrm{H}} 7.79-7.67\left(\mathrm{~m}, 9 \mathrm{H}, 4-\mathrm{ClC}_{6} \mathrm{H}_{4} \mathrm{~S}\right.$, $\left.\mathrm{C}_{6} \mathrm{H}_{5}\right), 6.56\left(\mathrm{~s}, 1 \mathrm{H}, \mathrm{H}_{P y r}^{4}\right), 5.76\left(\mathrm{~s}, 2 \mathrm{H}, \mathrm{NCH}_{2}\right), 3.65\left(\mathrm{t}, J 7.4 \mathrm{~Hz}, 2 \mathrm{H}, \mathrm{SCH}_{2}\right), 3.38$ (t, J $7.4 \mathrm{~Hz}$, $\left.2 \mathrm{H}, \mathrm{CH}_{2} \mathrm{CH}_{2} \mathrm{~S}\right) .{ }^{13} \mathrm{C}$ NMR $\left(100.61 \mathrm{MHz}, \mathrm{CDCl}_{3}\right): \delta_{\mathrm{C}} 150.8\left(\mathrm{C}_{\text {Pyr }}^{3}\right), 136.1\left(\mathrm{C}_{S}^{i}\right), 134.7\left(\mathrm{C}_{B n}^{i}\right)$, $132.1\left(\mathrm{C}^{4}\right), 130.9\left(\mathrm{C}^{3,5}\right), 129.3\left(\mathrm{C}_{P y r}^{5}\right), 129.0\left(\mathrm{C}^{2,6}{ }_{S}\right), 128.70\left(\mathrm{C}_{B n}^{3,5}\right), 127.9\left(\mathrm{C}_{B n}^{4}\right), 127.3$ $\left(\mathrm{C}^{2,6}{ }_{B n}\right), 104.1 \quad\left(\mathrm{C}_{P y r}^{4}\right), 52.6\left(\mathrm{NCH}_{2}\right), 33.5\left(\mathrm{SCH}_{2}\right), 28.7\left(\mathrm{CH}_{2} \mathrm{CH}_{2} \mathrm{~S}\right)$. Anal. Calcd for $\mathrm{C}_{18} \mathrm{H}_{16} \mathrm{Cl}_{2} \mathrm{~N}_{2} \mathrm{~S}$ : C, 59.51; H, 4.44; $\mathrm{Cl}, 19.52 ; \mathrm{N}, 7.71 ; \mathrm{S}, 8.83$. Found: $\mathrm{C}, 59.53 ; \mathrm{H}, 4.45 ; \mathrm{Cl}$, $19.50 ; \mathrm{N}, 7.69 ; \mathrm{S}, 8.85 \%$.

1-Benzyl-3-\{2-[(4-bromophenyl)sulfanyl]ethyl\}-5-chloro-1 $\boldsymbol{H}$-pyrazole (6bc). Obtained from vinylpyrazole $4 \mathbf{b}(0.22 \mathrm{~g})$ and 4-bromobenzenethiol $\mathbf{5 c}(0.19 \mathrm{~g})$. Colourless oil $(0.38 \mathrm{~g}, 93 \%)$, bp 288-292 ${ }^{\circ} \mathrm{C}(15 \mathrm{~mm} \mathrm{Hg}),{ }^{1} \mathrm{H}$ NMR $\left(400.13 \mathrm{MHz}, \mathrm{CDCl}_{3}\right): \delta_{\mathrm{H}}$ 7.86-7.66 (m, 9H, 4- $\mathrm{BrC}_{6} \mathrm{H}_{4} \mathrm{~S}$, $\left.\mathrm{C}_{6} \mathrm{H}_{5}\right), 6.56\left(\mathrm{~s}, 1 \mathrm{H}, \mathrm{H}_{P y r}^{4}\right), 5.76\left(\mathrm{~s}, 2 \mathrm{H}, \mathrm{NCH}_{2}\right), 3.65\left(\mathrm{t}, J 7.7 \mathrm{~Hz}, 2 \mathrm{H}, \mathrm{SCH}_{2}\right), 3.38(\mathrm{t}, J 7.7 \mathrm{~Hz}$, $\left.2 \mathrm{H}, \mathrm{C}_{2} \mathrm{CH}_{2} \mathrm{~S}\right) .{ }^{13} \mathrm{C}$ NMR $\left(100.61 \mathrm{MHz}, \mathrm{CDCl}_{3}\right): \delta_{\mathrm{C}} 150.8\left(\mathrm{C}^{3}{ }_{P y r}\right), 136.2\left(\mathrm{C}_{S}^{i}\right), 132.3\left(\mathrm{C}_{B n}^{i}\right)$, $132.0\left(\mathrm{C}^{2,6}{ }_{S}\right), 131.1\left(\mathrm{C}^{3,5}{ }_{S}\right), 129.5\left(\mathrm{C}_{P y r}^{5}\right), 128.8\left(\mathrm{C}_{B n}^{3,5}\right), 127.9\left(\mathrm{C}_{B n}^{4}\right), 127.3\left(\mathrm{C}^{2,6}{ }_{B n}\right), 120.0\left(\mathrm{C}_{S}^{4}\right)$, 
104.2 $\left(\mathrm{C}^{4}{ }_{P y r}\right), 52.7\left(\mathrm{NCH}_{2}\right), 33.4\left(\mathrm{SCH}_{2}\right), 28.7\left(\mathrm{CH}_{2} \mathrm{CH}_{2} \mathrm{~S}\right)$. Anal. Calcd for $\mathrm{C}_{18} \mathrm{H}_{16} \mathrm{BrClN}_{2} \mathrm{~S}: \mathrm{C}$, 53.02; H, 3.96; N, 6.87; S, 7.86. Found: C, 53.06; H, 3.97; N, 6.87; S, $7.84 \%$.

5-Chloro-1-(1-methylethyl)-3-[2-(phenylsulfanyl)ethyl]-1H-pyrazole (6ca). Obtained from vinylpyrazole $4 \mathbf{c}(0.17 \mathrm{~g})$ and benzenethiol 5a $(0.11 \mathrm{~g})$. Colourless oil $(0.27 \mathrm{~g}, 95 \%)$. IR $\left(v_{\max }\right.$, $\left.\mathrm{cm}^{-1}\right): 3130\left(=\mathrm{C}-\mathrm{H}_{\mathrm{Pyr}}\right), 3085,3076,3058\left(=\mathrm{C}-\mathrm{H}_{\mathrm{Ph}}\right), 2980,2933\left(\mathrm{CH}_{\mathrm{Alk}}\right), 1584,1514(\mathrm{C}=\mathrm{C}, \mathrm{C}=\mathrm{N})$. ${ }^{1} \mathrm{H}$ NMR $\left(400.13 \mathrm{MHz}, \mathrm{CDCl}_{3}\right): \delta_{\mathrm{H}} 7.19-7.13\left(\mathrm{~m}, 5 \mathrm{H}, \mathrm{C}_{6} \mathrm{H}_{5}\right), 6.02\left(\mathrm{~s}, 1 \mathrm{H}, \mathrm{H}^{4}{ }_{\text {Pyr }}\right.$ ), 4.58 (spt, $J 6.6$ $\mathrm{Hz}, 2 \mathrm{H}, \mathrm{NCH}$ ), 3.17 (t, $J 7.3 \mathrm{~Hz}, 2 \mathrm{H}, \mathrm{SCH}_{2}$ ), 2.90 (t, $J 7.3 \mathrm{~Hz}, 2 \mathrm{H}, \mathrm{C}_{2} \mathrm{CH}_{2} \mathrm{~S}$ ), 1.43 (d, J $6.6 \mathrm{~Hz}$, $\left.6 \mathrm{H}, 2 \mathrm{CH}_{3}\right) .{ }^{13} \mathrm{C}$ NMR $\left(100.61 \mathrm{MHz}, \mathrm{CDCl}_{3}\right): \delta_{\mathrm{C}} 150.4,\left(\mathrm{C}^{3}{ }_{p y r}\right), 136.4\left(\mathrm{C}^{i}\right), 129.6\left(\mathrm{C}^{2,6}\right), 129.0$ $\left(\mathrm{C}^{4}\right), 126.1\left(\mathrm{C}^{3,5}\right), 124.3\left(\mathrm{C}_{p y r}^{3}\right), 103.4\left(\mathrm{C}_{p y r}^{4}\right), 50.1(\mathrm{NCH}), 33.4\left(\mathrm{SCH}_{2}\right), 29.0\left(\underline{C H}_{2} \mathrm{CH}_{2} \mathrm{~S}\right), 22.1$ $\left(\underline{\mathrm{CH}}_{3}\right)$. Anal. Calcd for $\mathrm{C}_{14} \mathrm{H}_{17} \mathrm{ClN}_{2} \mathrm{~S}: \mathrm{C}, 59.88 ; \mathrm{H}, 6.10 ; \mathrm{Cl}, 12.62 ; \mathrm{N}, 9.98 ; \mathrm{S}, 11.42$. Found: $\mathrm{C}$, $59.53 ; \mathrm{H}, 6.17 ; \mathrm{Cl}, 12.69 ; \mathrm{N}, 10.12 ; \mathrm{S}, 11.33 \%$.

\section{5-Chloro-3-\{2-[(4-Chlorophenyl)sulfanyl]ethyl\}-1-(1-methylethyl)-1 $H$-pyrazole}

(6cb). Obtained from vinylpyrazole $4 \mathbf{c}(0.17 \mathrm{~g})$ and 4-chlorobenzenethiol $\mathbf{5 b}(0.14 \mathrm{~g})$. Colourless oil $(0.29 \mathrm{~g}, 95 \%)$. IR $\left(v_{\max }, \mathrm{cm}^{-1}\right): 3131\left(=\mathrm{C}-\mathrm{H}_{P y r}\right), 3104,3070\left(=\mathrm{C}-\mathrm{H}_{P h}\right), 2980,2933,2876\left(\mathrm{CH}_{\mathrm{Alk}}\right)$, 1578, $1513(\mathrm{C}=\mathrm{C}, \mathrm{C}=\mathrm{N}) .{ }^{1} \mathrm{H}$ NMR $\left(400.13 \mathrm{MHz}, \mathrm{CDCl}_{3}\right): \delta_{\mathrm{H}} 7.39-7.18\left(\mathrm{AA}^{\prime} \mathrm{BB}^{\prime}, J 8.7 \mathrm{~Hz}, 4 \mathrm{H}\right.$, $\left.\mathrm{C}_{6} \mathrm{H}_{4}\right), 6.00\left(\mathrm{~s}, 1 \mathrm{H}, \mathrm{H}_{P y r}^{4}\right), 4.59$ (spt, $\left.J 6.7 \mathrm{~Hz}, 2 \mathrm{H}, \mathrm{NCH}_{2}\right), 3.14\left(\mathrm{t}, J 7.3 \mathrm{~Hz}, 2 \mathrm{H}, \mathrm{SCH}_{2}\right), 2.87(\mathrm{t}, J$ $\left.7.3 \mathrm{~Hz}, 2 \mathrm{H}, \mathrm{C}_{2} \mathrm{CH}_{2} \mathrm{~S}\right), 1.43\left(\mathrm{~d}, J 6.7 \mathrm{~Hz}, 6 \mathrm{H}, 2 \mathrm{CH}_{3}\right) .{ }^{13} \mathrm{C} \mathrm{NMR}\left(100.61 \mathrm{MHz}, \mathrm{CDCl}_{3}\right): \delta_{\mathrm{C}} 150.1$ $\left(\mathrm{C}^{3}{ }_{p y r}\right), 134.9\left(\mathrm{C}^{i}\right), 132.1\left(\mathrm{C}^{4}\right), 130.9\left(\mathrm{C}^{3,5}\right), 129.1\left(\mathrm{C}^{2,6}\right), 125.7\left(\mathrm{C}^{5}{ }_{p y r}\right), 103.3\left(\mathrm{C}^{4}{ }_{p y r}\right), 50.1(\mathrm{NCH})$, $33.7\left(\mathrm{SCH}_{2}\right), 28.9\left(\underline{\mathrm{CH}}_{2} \mathrm{CH}_{2} \mathrm{~S}\right), 22.1\left(\mathrm{CH}_{3}\right)$. Anal. Calcd for $\mathrm{C}_{14} \mathrm{H}_{16} \mathrm{Cl}_{2} \mathrm{~N}_{2} \mathrm{~S}: \mathrm{C}, 53.34 ; \mathrm{H} .12 ; \mathrm{Cl}$, 22.49; N, 8.89; S, 10.17. Found: C, 53.28; H 5.03; Cl, 22.35; N, 8.69; S, $10.03 \%$.

5-Chloro-1-(3-methylbutyl)-3-[2-(phenylsulfanyl)ethyl]-1H-pyrazole (6da). Obtained from vinylpyrazole $4 \mathbf{d}(0.20 \mathrm{~g})$ and benzenethiol $5 \mathrm{a}(0.11 \mathrm{~g})$. Colourless oil $(0.30 \mathrm{~g}, 98 \%)$. IR $\left(v_{\max }\right.$, $\left.\mathrm{cm}^{-1}\right): 3131\left(=\mathrm{C}-\mathrm{H}_{P y r}\right), 3104,3069\left(=\mathrm{C}-\mathrm{H}_{P h}\right), 2980,2933,2876\left(\mathrm{CH}_{\mathrm{Alk}}\right), 1578,1513(\mathrm{C}=\mathrm{C}$, $\mathrm{C}=\mathrm{N}) .{ }^{1} \mathrm{H}$ NMR $\left(400.13 \mathrm{MHz}, \mathrm{CDCl}_{3}\right): \delta_{\mathrm{H}} 7.37-7.16\left(\mathrm{~m}, 5 \mathrm{H}, \mathrm{C}_{6} \mathrm{H}_{5}\right), 6.04\left(\mathrm{~s}, 1 \mathrm{H}, \mathrm{H}_{P y r}^{4}\right), 4.07(\mathrm{t}, J$ $\left.7.6 \mathrm{~Hz}, 2 \mathrm{H}, \mathrm{NCH}_{2}\right), 3.18$ (t, J 7.0 Hz, 2H, $\left.\mathrm{SCH}_{2}\right), 2.88$ (t, J 7.0 Hz, 2H, $\left.\underline{\mathrm{H}}_{2} \mathrm{CH}_{2} \mathrm{~S}\right), 1.69$ (m, $2 \mathrm{H}$, $\left.\mathrm{CH}_{2}\right), 1.61(\mathrm{~m}, 1 \mathrm{H}, \mathrm{CH}), 0.95\left(\mathrm{~d}, J 6.4 \mathrm{~Hz}, 6 \mathrm{H}, 2 \mathrm{CH}_{3}\right) .{ }^{13} \mathrm{C} \mathrm{NMR}\left(100.61 \mathrm{MHz}, \mathrm{CDCl}_{3}\right): \delta_{\mathrm{C}}$ 150.4, $\left(\mathrm{C}_{\text {pyr }}^{3}\right), 136.2\left(\mathrm{C}^{i}\right), 129.4\left(\mathrm{C}^{2,6}\right), 128.8\left(\mathrm{C}^{3,5}\right), 126.4\left(\mathrm{C}^{4}\right), 125.9\left(\mathrm{C}_{p y r}^{5}\right), 103.4\left(\mathrm{C}_{p y r}^{4}\right), 47.3$ $\left(\mathrm{NCH}_{2}\right), 38.5\left(\mathrm{CH}_{2}\right), 33.2\left(\mathrm{CH}_{2} \mathrm{~S}\right), 28.7\left(\mathrm{CH}_{2} \mathrm{CH}_{2} \mathrm{~S}\right), 25.6(\mathrm{CH}), 22.3\left(\mathrm{CH}_{3}\right) . \mathrm{MS}(\mathrm{EI}, 70 \mathrm{eV}), \mathrm{m} / \mathrm{z}$ (\%): $308\left(\mathrm{M}^{+}, 0.1\right), 286$ (46), 253 (15), 157 (52), 143 (80), 130 (100). Anal. Calcd for $\mathrm{C}_{16} \mathrm{H}_{21} \mathrm{ClN}_{2} \mathrm{~S}: \mathrm{C}, 62.22 ; \mathrm{H} 6.85 ; \mathrm{Cl}, 11.48 ; \mathrm{N}, 9.07 ; \mathrm{S}, 10.38$. Found: C, 62.17; H 6.73; Cl, 11.26; $\mathrm{N}, 9.01 ; \mathrm{S}, 10.33 \%$.

5-Chloro-1-heptyl-3-[2-(phenylsulfanyl)ethyl]-1H-pyrazole (6ea). Obtained from vinylpyrazole $4 \mathrm{e}(0.23 \mathrm{~g})$ and benzenethiol $5 \mathbf{a}(0.11 \mathrm{~g})$. Colourless oil $(0.33 \mathrm{~g}, 98 \%)$. IR $\left(v_{\max }, \mathrm{cm}^{-1}\right)$ : $3132\left(=\mathrm{C}-\mathrm{H}_{P y r}\right), 3071,3058\left(=\mathrm{C}-\mathrm{H}_{P h}\right), 2956,2928,2856\left(\mathrm{CH}_{\mathrm{Alk}}\right), 1584,1513(\mathrm{C}=\mathrm{C}, \mathrm{C}=\mathrm{N}) .{ }^{1} \mathrm{H}$ NMR (400.13 MHz, $\left.\mathrm{CDCl}_{3}\right): \delta_{\mathrm{H}} 7.33-7.11\left(\mathrm{~m}, 5 \mathrm{H}, \mathrm{C}_{6} \mathrm{H}_{5}\right), 5.99\left(\mathrm{~s}, 1 \mathrm{H}, \mathrm{H}^{4}{ }_{P y r}\right), 4.01(\mathrm{t}, J 7.4 \mathrm{~Hz}$, $\left.2 \mathrm{H}, \mathrm{NCH}_{2}\right), 3.14\left(\mathrm{t}, J 7.8 \mathrm{~Hz}, 2 \mathrm{H}, \mathrm{CH}_{2} \mathrm{~S}\right), 2.86\left(\mathrm{t}, J 7.8 \mathrm{~Hz}, 2 \mathrm{H}, \mathrm{C}_{2} \mathrm{CH}_{2} \mathrm{~S}\right), 1.25\left(\mathrm{~m}, 10 \mathrm{H}, 5 \mathrm{CH}_{2}\right)$, $0.85\left(\mathrm{t}, J 6.9 \mathrm{~Hz}, 3 \mathrm{H}, \mathrm{CH}_{3}\right) .{ }^{13} \mathrm{C} \mathrm{NMR}\left(100.61 \mathrm{MHz}, \mathrm{CDCl}_{3}\right): \delta_{\mathrm{C}} 150.6\left(\mathrm{C}^{3}{ }_{\text {pyr }}\right), 136.3\left(\mathrm{C}^{i}\right), 129.7$ $\left(\mathrm{C}^{2,6}\right), 129.0\left(\mathrm{C}^{3,5}\right), 126.8\left(\mathrm{C}^{4}\right), 126.2\left(\mathrm{C}^{5}{ }_{p y r}\right), 103.5\left(\mathrm{C}^{4}{ }_{\text {pyr }}\right), 49.1\left(\mathrm{NCH}_{2}\right), 33.5\left(\mathrm{CH}_{2} \mathrm{~S}\right), 31.8$ $\left(\mathrm{CH}_{2} \mathrm{CH}_{2} \mathrm{~S}\right), 30.0\left(\mathrm{CH}_{2}\right), 28.9\left(\mathrm{CH}_{2}\right), 28.9\left(\mathrm{CH}_{2}\right), 26.6\left(\mathrm{CH}_{2}\right), 22.7\left(\mathrm{CH}_{2}\right), 14.2\left(\mathrm{CH}_{3}\right) . \mathrm{MS}$ (EI, 70 $\mathrm{eV}), \mathrm{m} / \mathrm{z}$ (\%): 336 (M+63), 301 (92), 251 (24), 227 (37), 214 (30), 191 (16), 179 (22), 129 (67), 
123 (100). Anal. Calcd for $\mathrm{C}_{18} \mathrm{H}_{25} \mathrm{ClN}_{2} \mathrm{~S}: \mathrm{C}, 64.17 ; \mathrm{H}$ 7.48; Cl, 10.52; N, 8.31; S, 9.52. Found: $\mathrm{C}$, $64.12 ; \mathrm{H} 7.63 ; \mathrm{Cl}, 10.26 ; \mathrm{N}, 8.11 ; \mathrm{S}, 9.33 \%$.

5-Chloro-3-2-[(4-chlorophenyl)sulfanyl]ethyl-1-heptyl-1H-pyrazole (6eb). Obtained from vinylpyrazole $4 \mathbf{e}(0.23 \mathrm{~g})$ and 4-chlorobenzenethiol $5 \mathbf{b}(0.14 \mathrm{~g})$. Colourless oil $(0.35 \mathrm{~g}, 95 \%)$. IR $\left(v_{\max }, \mathrm{cm}^{-1}\right): 3133\left(=\mathrm{C}-\mathrm{H}_{P y r}\right), 3107,3070,3042\left(=\mathrm{C}-\mathrm{H}_{P h}\right), 2928,2856\left(\mathrm{CH}_{\mathrm{Alk}}\right), 1573,1513(\mathrm{C}=\mathrm{C}$, $\mathrm{C}=\mathrm{N}) .{ }^{1} \mathrm{H}$ NMR $\left(400.13 \mathrm{MHz}, \mathrm{CDCl}_{3}\right): \delta_{\mathrm{H}} 7.28,7.21\left(\mathrm{AA}^{\prime} \mathrm{BB}^{\prime}, J 8.7 \mathrm{~Hz}, 4 \mathrm{H}, \mathrm{C}_{6} \mathrm{H}_{4}\right), 6.01(\mathrm{~s}, 1 \mathrm{H}$, $\mathrm{H}_{\text {Pyr }}^{4}$ ), $4.04\left(\mathrm{t}, J 7.2 \mathrm{~Hz}, 2 \mathrm{H}, \mathrm{NCH}_{2}\right), 3.15\left(\mathrm{t}, J 7.4 \mathrm{~Hz}, 2 \mathrm{H}, \mathrm{CH}_{2} \mathrm{~S}\right), 2.87$ (t, J $7.4 \mathrm{~Hz}, 2 \mathrm{H}$, $\left.\mathrm{C}_{2} \mathrm{CH}_{2} \mathrm{~S}\right), 1.28\left(\mathrm{~m}, 10 \mathrm{H}, 5 \mathrm{CH}_{2}\right), 0.87\left(\mathrm{t}, J 7.4 \mathrm{~Hz}, 3 \mathrm{H}, \mathrm{CH}_{3}\right) .{ }^{13} \mathrm{C} \mathrm{NMR}\left(100.61 \mathrm{MHz}, \mathrm{CDCl}_{3}\right)$ : $\delta_{\mathrm{C}} 150.2\left(\mathrm{C}^{3}{ }_{p y r}\right), 134.8\left(\mathrm{C}^{i}\right), 132.1\left(\mathrm{C}^{4}\right), 130.9\left(\mathrm{C}^{3,5}\right), 129.0\left(\mathrm{C}^{2,6}\right), 126.7\left(\mathrm{C}_{p y r}^{5}\right), 103.4\left(\mathrm{C}_{p y r}^{4}\right)$, $48.9\left(\mathrm{NCH}_{2}\right), 33.6\left(\mathrm{CH}_{2} \mathrm{~S}\right), 31.7\left(\mathrm{CH}_{2} \mathrm{CH}_{2} \mathrm{~S}\right), 29.9\left(\mathrm{CH}_{2}\right), 28.8\left(\mathrm{CH}_{2}\right), 28.7\left(\mathrm{CH}_{2}\right), 26.4\left(\mathrm{CH}_{2}\right)$, $22.6\left(\mathrm{CH}_{2}\right), 14.1\left(\mathrm{CH}_{3}\right)$. MS (EI, $\left.70 \mathrm{eV}\right), \mathrm{m} / \mathrm{z}(\%): 370\left(\mathrm{M}^{+}, 74\right), 335$ (97), 285 (25), 227 (42), 214 (58), 157 (72), 129 (100).Anal. Calcd for $\mathrm{C}_{18} \mathrm{H}_{24} \mathrm{Cl}_{2} \mathrm{~N}_{2} \mathrm{~S}: \mathrm{C}, 58.22 ; \mathrm{H}, 6.51 ; \mathrm{Cl}, 19.09 ; \mathrm{N}$, 7.54; S, 8.63. Found: C, 58.14; H, 6.69; Cl, 19.26; N, 7.31; S, $8.43 \%$.

5-Chloro-1-methyl-3-[1-methyl-2-(phenylsulfanyl)ethyl]-1H-pyrazole (6fa). Obtained from vinylpyrazole $4 \mathbf{f}(0.16 \mathrm{~g})$ and benzenethiol $5 \mathrm{a}(0.11 \mathrm{~g})$. Colourless oil, $(0.26 \mathrm{~g}, 97 \%)$. IR ( $v_{\max }$, $\left.\mathrm{cm}^{-1}\right): 3135\left(=\mathrm{C}-\mathrm{H}_{P y r}\right), 3110,3072,3045\left(=\mathrm{C}-\mathrm{H}_{P h}\right), 2927,2860\left(\mathrm{CH}_{\mathrm{Alk}}\right), 1570,1515(\mathrm{C}=\mathrm{C}$, $\mathrm{C}=\mathrm{N}) .{ }^{1} \mathrm{H}$ NMR $\left(400.13 \mathrm{MHz}, \mathrm{CDCl}_{3}\right): \delta_{\mathrm{H}} 7.37-7.16\left(\mathrm{~m}, 5 \mathrm{H}, \mathrm{C}_{6} \mathrm{H}_{5}\right), 6.05\left(\mathrm{~s}, 1 \mathrm{H}, \mathrm{H}_{P y r}^{4}\right), 3.78(\mathrm{~s}$, $\left.3 \mathrm{H}, \mathrm{NCH}_{3}\right), 3.30(\mathrm{~m}, 1 \mathrm{H}, \mathrm{CH}), 3.05\left(\mathrm{~m}, 2 \mathrm{H}, \mathrm{CH}_{2} \mathrm{~S}\right), 1.37\left(\mathrm{~d}, J 6.6 \mathrm{~Hz}, 3 \mathrm{H}, \mathrm{CH}_{3}\right)$. Anal. Calcd for $\mathrm{C}_{13} \mathrm{H}_{15} \mathrm{ClN}_{2} \mathrm{~S}$ : C, 58.53; H, 5.67; Cl, 13.29; N, 10.50; S, 12.02. Found: C, 58.17; H, 5.43; Cl, $13.20 ; \mathrm{N}, 10.31 ; \mathrm{S}, 12.13 \%$.

5-Chloro-3-\{2-[(4-chlorophenyl)sulfanyl]-1-methylethyl\}-1-methyl-1 H-pyrazole

(6fb). Obtained from vinylpyrazole $\mathbf{4 f}(0.16 \mathrm{~g})$ and 4-chlorobenzenethiol $\mathbf{5 b}(0.14 \mathrm{~g})$. Colourless oil $(0.29 \mathrm{~g}, 98 \%)$. IR $\left(v_{\max }, \mathrm{cm}^{-1}\right): 3133\left(=\mathrm{C}-\mathrm{H}_{P y r}\right), 3108,3070,3043\left(=\mathrm{C}-\mathrm{H}_{P h}\right), 2981,2934,2860$ $\left(\mathrm{CH}_{\mathrm{Alk}}\right), 1573,1513(\mathrm{C}=\mathrm{C}, \mathrm{C}=\mathrm{N}) .{ }^{1} \mathrm{H}$ NMR $\left(400.13 \mathrm{MHz}, \mathrm{CDCl}_{3}\right): \delta_{\mathrm{H}} 7.21-7.13\left(\mathrm{AA}^{\prime} \mathrm{BB}^{\prime}, 4 \mathrm{H}\right.$, $\left.\mathrm{C}_{6} \mathrm{H}_{4}\right), 5.95\left(\mathrm{~s}, 1 \mathrm{H}, \mathrm{H}^{4}{ }_{P y r}\right), 3.69\left(\mathrm{~s}, 3 \mathrm{H}, \mathrm{NCH}_{3}\right), 3.18(\mathrm{~m}, 1 \mathrm{H}, \mathrm{CH}), 2.95\left(\mathrm{~m}, 2 \mathrm{H}, \mathrm{CH}_{2} \mathrm{~S}\right), 1.28(\mathrm{~d}, J$ $6.5 \mathrm{~Hz}, 3 \mathrm{H}, \mathrm{CH}_{3}$ ). Anal. Calcd for $\mathrm{C}_{13} \mathrm{H}_{14} \mathrm{Cl}_{2} \mathrm{~N}_{2} \mathrm{~S}: \mathrm{C}, 51.83 ; \mathrm{H}, 4.68 ; \mathrm{Cl}, 23.54 ; \mathrm{N}, 9.30 ; \mathrm{S}, 10.64$. Found: C, 51.67; H, 4.53; Cl, 23.26; N, 9.11; S, $10.46 \%$.

5-Chloro-3-\{2-[(4-chlorophenyl)sulfanyl]propyl\}-1-methyl-1H-pyrazole (6gb). Obtained from vinylpyrazole $4 \mathrm{~g}(0.16 \mathrm{~g})$ and 4-chlorobenzenethiol $\mathbf{5 b}(0.14 \mathrm{~g})$. Colourless oil $(0.29 \mathrm{~g}$, 96\%). IR $\left(v_{\max }, \mathrm{cm}^{-1}\right): 3131\left(=\mathrm{C}-\mathrm{H}_{P y r}\right), 3110,3072,3040\left(=\mathrm{C}-\mathrm{H}_{P h}\right), 2983,2931,2856\left(\mathrm{CH}_{\mathrm{Alk}}\right)$, 1575, $1515(\mathrm{C}=\mathrm{C}, \mathrm{C}=\mathrm{N}) .{ }^{1} \mathrm{H}$ NMR $\left(400.13 \mathrm{MHz}, \mathrm{CDCl}_{3}\right): \delta_{\mathrm{H}} 7.26-7.13\left(\mathrm{AA}^{\prime} \mathrm{BB}^{\prime}, 4 \mathrm{H}, \mathrm{C}_{6} \mathrm{H}_{4}\right)$, $5.97\left(\mathrm{~s}, 1 \mathrm{H}, \mathrm{H}_{\text {Pyr }}^{4}\right), 3.69\left(\mathrm{~s}, 3 \mathrm{H}, \mathrm{NCH}_{3}\right), 3.39(\mathrm{~m}, 1 \mathrm{H}, \mathrm{CH}), 2.81,2.63\left(\mathrm{~m}, 2 \mathrm{H}, \mathrm{CH}_{2}\right), 1.21(\mathrm{~d}, J 6.6$ $\mathrm{Hz}, 3 \mathrm{H}, \mathrm{CH}_{3}$ ). Anal. Calcd for $\mathrm{C}_{13} \mathrm{H}_{14} \mathrm{Cl}_{2} \mathrm{~N}_{2} \mathrm{~S}: \mathrm{C}, 51.83 ; \mathrm{H}, 4.68 ; \mathrm{Cl}, 23.54 ; \mathrm{N}, 9.30 ; \mathrm{S}, 10.64$. Found: C, 51.30.17; H 4.37; Cl, 23.18; N, 9.14; S, $10.38 \%$.

5-Chloro-1-(1-methylethyl)-3-[1-(phenylsulfanyl)ethyl]-1H-pyrazole $\quad$ (7). Potassium hydroxide ( $5 \mathrm{mmol}, 0.30 \mathrm{~g})$ and benzenethiol $5 \mathbf{5}(1.5 \mathrm{mmol}, 0.17 \mathrm{~g})$ was stirred for $20 \mathrm{~min}$ in DMSO $(1.5 \mathrm{~mL})$. Then pyrazole $\mathbf{3 d}(1.5 \mathrm{mmol}, 0.31 \mathrm{~g})$ was added and the reaction mixture was stirred for $5 \mathrm{~h}$ at $\mathrm{rt}$. A mixture was poured into water and extracted with dichloromethane $(3 \times 10$ $\mathrm{mL}$ ). The extract was dried over $\mathrm{MgSO}_{4}$, dichloromethane was evaporated to give the target pyrazole derivative 7 as yellow oil $(0.35 \mathrm{~g}, 82 \%)$. ${ }^{1} \mathrm{H} \mathrm{NMR}\left(400.13 \mathrm{MHz}, \mathrm{CDCl}_{3}\right): \delta_{\mathrm{H}} 7.32-717$ 
$\left(\mathrm{m}, 5 \mathrm{H}, \mathrm{C}_{6} \mathrm{H}_{5}\right), 6.13\left(\mathrm{~s}, 1 \mathrm{H}, \mathrm{H}_{\text {Pyr }}^{4}\right), 4.56(\mathrm{~m}, \mathrm{~J}$ 6.6, $6.7 \mathrm{~Hz}, 1 \mathrm{H}, \mathrm{NCH}), 4.44(\mathrm{q}, \mathrm{J} 7.0 \mathrm{~Hz}, 1 \mathrm{H}$, CHS), 1.58 (d, J 7.0 Hz, 3H, CH $), 1.40$ (d, J $\left.6.6 \mathrm{~Hz}, 3 \mathrm{H}, \mathrm{CH}_{3}\right), 1.34$ (d, J $\left.6.7 \mathrm{~Hz}, 3 \mathrm{H}, \mathrm{CH}_{3}\right) .{ }^{13} \mathrm{C}$ NMR (100.61 MHz, $\left.\mathrm{CDCl}_{3}\right): \delta_{\mathrm{C}} 153.8\left(\mathrm{C}_{p y r}^{3}\right), 135.2\left(\mathrm{C}^{i}\right), 132.3\left(\mathrm{C}^{2,6}\right), 128.7\left(\mathrm{C}^{3,5}\right), 127.1\left(\mathrm{C}^{5}{ }_{p y r}\right)$, $125.8\left(\mathrm{C}^{4}\right), 102.1\left(\mathrm{C}^{4}{ }_{\text {pyr }}\right), 50.2(\mathrm{NCH}), 41.6(\mathrm{CHS}), 22.1,21.0,21.2\left(\mathrm{CH}_{3}\right) . \mathrm{MS}, \mathrm{m} / \mathrm{z}(\%) 280$ (M+, 12), 171 (84), 129 (100), 109 (11). Anal. Calcd for $\mathrm{C}_{15} \mathrm{H}_{17} \mathrm{ClN}_{2} \mathrm{~S}: \mathrm{C}, 59.88 ; \mathrm{H}, 6.10 ; \mathrm{Cl}$, $12.62 ; \mathrm{N}, 9.98$. Found: $\mathrm{C}, 59.95 ; \mathrm{H}, 6.07 ; \mathrm{Cl}, 12.25 ; \mathrm{N}, 9.77 \%$.

\section{References}

1. Fustero, S.; Sanchez-Rosello, M.; Barrio, P.; Simon-Fuentes, A. Chem. Rev. 2011, 111, 6984. http://dx.doi.org/10.1021/cr2000459

2. Janin, Y. L. Chem. Rev. 2012, 112, 3924. http://dx.doi.org/10.1021/cr200427q

3. Pérez-Fernández, R.; Goya, P.; Elguero, J. Arkivoc 2014, (ii), 233. http://dx.doi.org/10.3998/ark.5550190.p008.131

4. Ivachtchenko, A. V. Russ. Chem. Rev. 2014, 83, 439. http://dx.doi.org/10.1070/RC2014v083n05ABEH004371

5. Pizzuti, L.; Barschak, A. G.; Stefanello, F. M.; Farias, M. D.; Lencina, C.; Roesch-Ely, M.; Cunico, W.; Moura, S.; Pereira, C. M. P. Curr. Org. Chem. 2014, 18, 115. http://dx.doi.org/10.2174/13852728113179990029

6. Elguero, J.; Goya, P.; Jagerovic, N.; Silva, A. M. S. In Targets in Heterocyclic Systems. Chemistry and Properties, Vol. 6, Attanasi, O. A.; Spinelli, D., Eds.; Italian Soc. Chem. 2003, 53.

7. Genin, M. J.; Biles, C.; Keiser, B. J.; Poppe, S. M.; Swaney, S. M.; Tarpley, W. G.; Yagi, Y.; Romeo, D. L. J. Med. Chem. 2000, 43, 1034. http://dx.doi.org/10.1021/jm990383f

8. Bailey, D. M.; Hansen, P. E.; Hlavac, A. G.; Baizman, E. R.; Pearl, J.; DeFelice, A. F.; Feigenson, M. E. J. Med. Chem. 1985, 28, 256. http://dx.doi.org/10.1021/jm00380a020

9. Szabo, G.; Fischer, J.; Kis-Varga, A.; Gyires, K. J. Med. Chem. 2008, 51, 142. http://dx.doi.org/10.1021/jm070821f

10. Farag, A. M.; Mayhoub, A. S.; Barakat, S. E.; Bayomi, A. H. Bioorg. Med. Chem. 2008, 16, 881.

http://dx.doi.org/10.1016/j.bmc.2007.10.015

11. Vicentini, C. B.; Romagnoli, C.; Andreotti, E.; Mares, D. J. Agric. Food Chem. 2007, 55, 10331. http://dx.doi.org/10.1021/jf072077d

12. Li, Y.; Zhang, H.-Q.; Liu, J.; Yang, X.-P.; Liu, Z.-J. J. Agric. Food Chem. 2006, 54, 3636. http://dx.doi.org/10.1021/jf060074f 
13. Theodoridis, G. In Modern Crop Protection Compounds, Vol. 1, Kramer, W., Schirmer, U., Eds.; Wiley-VCH: Weinheim, 2007; p153.

14. Shiga, Y.; Okada, I.; Ikeda, Y.; Takizawa, E.; Fukuchi, T. J. Pesticide Sci. 2003, 28, 313. http://dx.doi.org/10.1584/jpestics.28.313

15. Grapov, A. F. Russ. Chem. Rev. 1999, 68, 697. http://dx.doi.org/10.1070/RC1999v068n08ABEH000510

16. Cristodoulou, M. S.; Kasiotis, K. M.; Fokialakis, N.; Tillitu, I.; Haroutounian, S. A. Tetrahedron Lett. 2008, 49, 7100. http://dx.doi.org/10.1016/j.tetlet.2008.09.098

17. Aajoud, A.; Ravanel, P.; Tissut, M. J. Agric. Food Chem. 2003, 51, 1347. http://dx.doi.org/10.1021/jf025843j

18. Potapov, A. S.; Domina, G. A.; Petrenko, T. V.; Khlebnikov, A. I. Polyhedron 2012, 33(1), 150. http://dx.doi.org/10.1016/j.poly.2011.11.039

19. Ojwach, S. O.; Darkwa, J. Inorg. Chim. Acta. 2011, 363, 1947. http://dx.doi.org/10.1016/j.ica.2010.02.014

20. Yang, L.; Okuda, F.; Kobayashi, K.; Nozaki, K.; Tanabe, Y.; Ishii, Y.; Haga, M. Inorg. Chem. 2008, 47, 7154. http://dx.doi.org/10.1021/ic800196s

21. Türkoglu, G.; Ulldemolins, C. P.; Müller, R.; Hübner, E.; Heinemann, F. W.; Wolf, M.; Burzlaff, N. Eur. J. Inorg. Chem. 2010, 2962. http://dx.doi.org/10.1002/ejic.201000115

22. Glenn, R. W.; Lim, M. US 20070050923 (2007); Chem. Abstr. 2007, 146, 322831.

23. Catalan, J.; Fabero, F.; Claramunt, R. M.; Santa Maria, M. D.; Concepcion F.-F.; Hernandez, F.; Cano, F. H.; Martinez-Ripoll, M.; Elguero, J.; Sastre, R. J. Amer. Chem. Soc. 1992, 114(13),5039. http://dx.doi.org/10.1021/ja00039a014

24. Cavero, E.; Uriel, S.; Romero, P.; Serrano, J. L.; Gimenez, R. J. Am. Chem. Soc. 2007, 129, 11608. http://dx.doi.org/10.1021/ja073639c

25. Thaker, B. T.; Solanki, D. B.; Patel, B. S.; Vansadia, A. D.; Dhimmar, Y. T. Molecular Crystals and Liquid Crystals 2012, 552(1), 134-146. http://dx.doi.org/10.1080/15421406.2011.609043

26. Ovcharenko, V. I.; Maryunina, K. Yu.; Fokin, S. V.; Tretyakov, E. V.; Romanenko, G. V.; Ikorskii, V. N. Russ. Chem. Bull. 2004, 11, 2406 http://dx.doi.org/10.1007/s11172-005-0136-4

27. Fokin, S.; Ovcharenko, V.; Romanenko, G.; Ikorskii, V. Inorg.Chem. 2004, 43, 969. http://dx.doi.org/10.1021/ic034964d

28. Levkovskaya, G. G.; Kobelevskaya, V. A.; Rudyakova, E. V.; Ha, Q. K.; Samultsev, D. O.; Rozentsveig, I. B. Tetrahedron 2011, 67(10), 1844. 
http://dx.doi.org/10.1016/j.tet.2011.01.028

29. Han, L. M.; Timmons,R. B.; Dariusz, B.; Pielichowski J. Chem. Mater. 1998, 10, 1422. http://dx.doi.org/10.1021/cm970779k

30. Gai, X.; Grigg, R.; Sridharan, R.; Collard, S.; Muir, J. E. J. Chem. Soc. Chem. Comm. 2000, 2053.

http://dx.doi.org/10.1039/B005452F

31. Domnina, E. S.; Es'kova, L. A.; Petrova, E. V.; Chipanina, N. N.; Voronov, V. K.; Afonin, A. V.; Skvortsova, G. G. Zh. Neorg. Khim. 1987, 32, 1523. Chem. Abstr. 1987, 107, 189498.

32. Es'kova, L. A.; Erushnikova, L. P.; Afonin, A. V.; Domnina, E. S. Russ. Chem. Bull. 1992, 1462.

http://dx.doi.org/10.1007/BF00864347

33. Diaz-Ortiz, A.; Carrillo, J. R.; Diez-Barra, E.; de la Hoz, A.; Gomez-Escalonilla, M.

Tetrahedron 1996, 52, 9237. http://dx.doi.org/10.1016/0040-4020(96)00473-5

34. Diaz-Ortiz, A.; de la Hoz, A.; Langa, F. Green Chem. 2000, 2, 165. http://dx.doi.org/10.1039/B003752O

35. Simon, M. M.; Sepulveda, J. Tetrahedron 1986, 42, 6683. http://dx.doi.org/10.1016/S0040-4020(01)82108-6

36. Carrillo, J. R.; Diaz-Ortiz, A.; Hoz, A.; Gomez-Escalonilla, M. J.; Moreno, A.; Prieto, P. Tetrahedron 1999, 55, 9623. http://dx.doi.org/10.1016/S0040-4020(99)00508-6

37. Haneda, A.; Imagawa, T.; Kawanici, M. Bull. Chem. Soc. Jpn. 1976, 49, 748. http://dx.doi.org/10.1246/bcsj.49.748

38. Tomilov, Yu. V.; Shulishov, E. V.; Kostitsyn, A. B.; Nefedov, O. M. Russ. Chem. Bull. 1994, 43,612 . http://dx.doi.org/10.1007/BF00699834

39. Rudyakova, E. V.; Samultsev, D. O.; Levkovskaya, G. G. Russ. J. Org. Chem. 2012, 48, 1388 http://dx.doi.org/10.1134/S1070428012100259

40. Rudyakova, E. V.; Samultsev, D. O.; Levanova, E. P.; Levkovskaya, G. G. Russ. J. Org. Chem. 2013, 49, 734. http://dx.doi.org/10.1134/S1070428013050175

41. Maximova, Marina A. ( $\mathrm{PhD})$. Synthesis and reactivity of $\mathrm{N}$-izopropenilazolov: thesis abstract on scientific degree of candidate of chemical sciences; [Irkutsk, Institute of Chemistry, 2007].

42. Skvortsova, G. G.; Glaskova, N. P.; Domnina, E.S.; Voronov, V. K. Chem. Heterocycl. Compd. 1970, 2, 153. http://dx.doi.org/10.1007/BF00474986

43. Aliev, I. F.; Mikhaleva, A. I.; Gasanov, B. R. Chem. Heterocycl. Compd. 1990, 6, 624. http://dx.doi.org/10.1007/BF00756411 
44. Hussain, S.; Bharadwaj, S. K.; Chaudhury, M. K.; Kalita, H. Eur. J. Org. Chem. 2007, 2, 374.

http://dx.doi.org/10.1002/ejoc.200600691

45. Rajabi, F.; Saidi, M.R. J. Sulfur Chem. 2005, 26, 251.

http://dx.doi.org/10.1080/17415990500276279 